\title{
A BSDE Approach to Stochastic Differential Games with Regime Switching
}

\author{
J. Y. Li $^{1}$ and M. N. Tang (iD ${ }^{2}$ \\ ${ }^{1}$ College of Science, University of Shanghai for Science and Technology, Shanghai 200433, China \\ ${ }^{2}$ Department of Mathematics, Huzhou University, Huzhou, Zhejiang 313000, China
}

Correspondence should be addressed to M. N. Tang; tmorning@zjhu.edu.cn

Received 19 March 2021; Revised 6 April 2021; Accepted 17 April 2021; Published 10 June 2021

Academic Editor: Rongwei Guo

Copyright (c) 2021 J. Y. Li and M. N. Tang. This is an open access article distributed under the Creative Commons Attribution License, which permits unrestricted use, distribution, and reproduction in any medium, provided the original work is properly cited.

In this paper, we study a two-player zero-sum stochastic differential game with regime switching in the framework of forwardbackward stochastic differential equations on a finite time horizon. By means of backward stochastic differential equation methods, in particular that of the notion from stochastic backward semigroups, we prove a dynamic programming principle for both the upper and the lower value functions of the game. Based on the dynamic programming principle, the upper and the lower value functions are shown to be the unique viscosity solutions of the associated upper and lower Hamilton-Jacobi-Bellman-Isaacs equations.

\section{Introduction}

The differential game is concerned with the problem that multiple players make decisions, according to their own advantages and trade-off with other partners in a dynamic system. Stochastic differential games (SDGs) have been well studied. Recently, Lv [1] studied the two-player zero-sum SDGs in a regime switching model with an infinite horizon. Compared with the traditional diffusion model, the regime switching model has two obvious advantages. First, the underlying Markov chain can be used to model discrete events with larger long-term system impact. For instance, in financial markets, it is easy to capture market trend by using finite state Markov chain. However, it is difficult to incorporate this dynamic into pure diffusion model. Second, when conducting numerical experiments, regime switching models require very limited data input. In recent years, due to the capacity for characterizing all kinds of random events and the tractability, regime switching models have attracted extensive attention [1-3]. In this paper, we introduce a new method, which is different from the method in [1]. We investigate two-player zero-sum SDGs with regime switching on a finite time horizon by using the backward stochastic differential equation (BSDE) methods.

Pardoux and Peng [4] first introduced the nonlinear BSDEs in 1990. The theory of BSDE was originally developed by Peng [5] for stochastic control theory. And later Hamadène and Lepeltier [6] and Hamadène et al. [7] introduced this theory to SDGs. Buckdahn and Li [8] studied a recursive SDG problem and interpreted the relationship between the controlled system and the Hamilton-Jacobi-Bellman-Isaacs (HJBI) equation. The theory of BSDEs has been well studied and applied to many fields, such as stochastic control, SDGs, mathematical finance, and partial differential equation theory (see [5-7, 9-11] for details). The readers interested in other topics about game theory are referred to [12-15].

In this paper, let $(\Omega, \mathscr{F}, \mathbb{P})$ be a fixed probability space on which a d-dimensional Brownian motion $\left\{B_{s}\right\}_{s \in[0, T]}$ and a Markov chain $\left\{\theta_{s}\right\}_{s \in[0, T]}$ are defined on some sample space $\Omega$, $\mathscr{F}$ is the completed Borel $\sigma$-algebra over $\Omega$, and $P$ is the Wiener measure. Here, we assume that $\mathscr{F}=\left(\mathscr{F}_{s}\right)_{s \in[0, T]}$, where $\mathscr{F}_{S} \triangleq \mathscr{F}_{S}^{\theta} \vee \mathscr{F}_{S}^{B}$. Let $\left(\mathscr{F}_{S}^{B}\right)_{s \in[0, T]}$ denote the filtration 
generated by Brownian motion $\left\{B_{s}\right\}_{s \in[0, T]}$. And $\left(\mathscr{F}_{s}^{\theta}\right)_{s \in[0, T]}$ denote the filtration generated by the Markov chain $\left\{\theta_{s}\right\}_{s \in[0, T]}$. Assume that $B$. and $\theta$. are independent. The Markov chain $\left\{\theta_{s}\right\}_{s \in[0, T]}$ takes values in a finite state space $\mathscr{M}=\{1, \ldots, m\}$ and is observable. And the generator $Q=$ $\left(q_{i k}\right)_{i, k \in \mathscr{M}} \in R^{m \times m}$ of the Markov chain $\left\{\theta_{s}\right\}_{s \in[0, T]}$ is given by $P\left(\theta_{s+\delta}=k \mid \theta_{s}=i, s \in[0, T]\right)= \begin{cases}q_{i k} \delta+o(\delta), & \text { if } k \neq i, \\ 1+q_{i i} \delta+o(\delta), & \text { if } k=i,\end{cases}$

(1) where $q_{i k}$ is the transition rate from market regime $i$ to $k$, $q_{i i}=-\sum_{k \neq i} q_{i k}<0$, and $q_{i k} \geq 0$, for every $(i, k) \in \mathscr{M} \times \mathscr{M}$.

We will investigate a two-player zero-sum SDG with regime switching in the framework of BSDE on a finite time horizon. The dynamics of the SDG are described by the following functional stochastic differential equation (SDE): for $t \in[0, T]$,

$$
\begin{cases}\mathrm{d} X_{s}^{t, x, i ; u, v}=b\left(s, X_{s}^{t, x, i ; u, v}, \theta_{s}^{t, i}, u_{s}, v_{s}\right) \mathrm{d} s+\sigma\left(s, X_{s}^{t, x, i ; u, v}, \theta_{s}^{t, i}, u_{s}, v_{s}\right) \mathrm{d} B_{s}, & s \in[t, T] \\ X_{t}^{t, x, i ; u, v}=x, & \theta_{t}^{t, i}=i\end{cases}
$$

where $T>0$ is a fixed finite time horizon, $(t, x, i) \in[0, T] \times$ $R^{n} \times \mathscr{M}$ is regarded as the initial state, and $P\left\{\theta_{t}^{t, i}=i\right\}=1 . X_{t}$ is the value of $X$ at time $t$, and $(u, v)=\left(u_{s}, v_{s}\right)_{s \in[t, T]}$ are the pair of $\mathscr{F}_{t}$-adapted processes, take their values in some compact metric spaces $U$ and $V$, and are called admissible controls of the two players I and II, respectively. Precise assumptions on the coefficients $b$ and $\sigma$ are given in the next section.

The cost functional is introduced by BSDE:

$$
\begin{aligned}
Y_{s}^{t, x, i ; u, v}= & \Phi\left(X_{T}^{t, x, i ; u, v}, \theta_{T}^{t, i}\right) \\
& +\int_{s}^{T} f\left(r, X_{r}^{t, x, i ; u, v}, Y_{r}^{t, x, i ; u, v}, Z_{r}^{t, x, i ; u, v}, \theta_{r}^{t, i}, u_{r}, v_{r}\right) \mathrm{d} r-\int_{s}^{T} Z_{r}^{t, x, i, u, v} \mathrm{~d} B_{r}, \\
& s \in[t, T],
\end{aligned}
$$

where $X^{t, x, i ; u, v}$ and $\theta^{t, i}$ are introduced in (2). The above BSDE has a unique solution $\left(Y_{s}^{t, x, i ; u, v}, Z_{s}^{t, x, i ; u, v}\right)_{s \in[t, T]}$. And for given control processes $u \in U_{t, T}$ and $v \in V_{t, T}$, we introduce the associated cost functional:

$$
J(t, x, i ; u, v):=E\left[Y_{t}^{t, x, i ; u, v} \mid \mathscr{F}_{t}\right], \quad(t, x, i) \in[0, T] \times R^{n} \times \mathscr{M},
$$

where $Y^{t, x, i ; u, v}$ is defined by BSDE (3). In the game, player I aims to maximize (4) and contrarily player II aims to minimize (4). We define the lower and the upper value functions $W$ and $U$, respectively:

$$
\begin{gathered}
W(t, x, i):=\underset{\beta \in \mathscr{B}_{t, T}}{\operatorname{essinf}} \operatorname{esssup}_{u \in U_{t, T}} J(t, x, i ; u, \beta(u)), \\
U(t, x, i):=\underset{\alpha \in \mathscr{A}_{t, T}}{\operatorname{esssup}} \operatorname{essinf}_{v \in V_{t, T}} J(t, x, i ; \alpha(v), v) .
\end{gathered}
$$

Precise definitions of $\alpha$ and $\beta$ are given in the next section. In the case $W=U$ we say that the game admits a value. The main objective of this paper is to show that $W$ and $U$ are, respectively, the unique viscosity solutions of the following lower and upper HJBI equations, and both are systems consisting of $m$ coupled equations:

$$
\begin{aligned}
& \left\{\begin{array}{l}
\frac{\partial}{\partial t} W(t, x, i)+H^{-}\left(t, x, i, W, D W, D^{2} W\right)+\sum_{k \neq i} q_{i k}[W(t, x, k)-W(t, x, i)]=0 \\
W(T, x, i)=\Phi(x, i),
\end{array}\right. \\
& \left\{\begin{array}{l}
\frac{\partial}{\partial t} U(t, x, i)+H^{+}\left(t, x, i, U, D U, D^{2} U\right)+\sum_{k \neq i} q_{i k}[U(t, x, k)-U(t, x, i)]=0 \\
U(T, x, i)=\Phi(x, i),
\end{array}\right.
\end{aligned}
$$


associated with

$$
\begin{aligned}
& H^{-}(t, x, i, r, p, A, u, v)=\sup _{u \in U} \inf _{v \in V} H(t, x, i, r, p, A, u, v), \\
& H^{+}(t, x, i, r, p, A, u, v)=\inf _{v \in V} \sup _{u \in U} H(t, x, i, r, p, A, u, v),
\end{aligned}
$$

where $H$ is defined as

$$
\begin{aligned}
H(t, x, i, p, A, u, v)= & \frac{1}{2} \operatorname{Tr}\left(\sigma \sigma^{T}(t, x, i, u, v) \cdot A\right)+\langle b(t, x, i, u, v), p\rangle \\
& +f\left(t, x, i, r, \sigma^{T}(t, x, i, u, v) p, u, v\right),
\end{aligned}
$$

for $(t, x, i, r, p, A, u, v) \in[0, T] \times R^{n} \times \mathscr{M} \times R \times R^{n} \times R^{n \times n}$ $\times U \times V \longrightarrow R$. If Isaacs' condition holds, i.e., $H^{-}=H^{+}$, then (5) and (6) coincide, and the uniqueness of viscosity solution implies $W=U$, that is, the game admits a value.

The paper is organized as follows. In Section 2, we introduce some notations and preliminaries, which will be needed in what follows. In Section 3, we introduce the dynamic programming principle. In Section 4, based on the dynamic programming principle, we investigate that the upper and the lower value functions are the unique viscosity solutions of the associated upper and lower HJBI equations.

\section{Preliminaries}

Let us introduce the following spaces, which will be needed in what follows.

$$
\begin{aligned}
L^{2}\left(\Omega, \mathscr{F}_{T}, P ; R^{n}\right) ; & =\left\{\xi: \Omega \longrightarrow R^{n} \text { is } \mathscr{F}_{T}-\text { measurable variable such that } E\left[|\xi|^{2}\right]<+\infty\right\}, \\
\mathcal{S}^{2}\left(0, T ; \mathrm{R}^{n}\right) & :=\left\{(\psi(t))_{t \in[0, T]} \mathrm{R}^{n}-\text { valued } \mathscr{F}_{T}^{\theta} \vee \mathscr{F}^{B}-\text { adapted continous process: } E\left[\sup _{t \in[0, T]}|\psi(t)|^{2}\right]<+\infty\right\}, \\
\mathscr{H}^{2}\left(0, T ; \mathrm{R}^{n}\right) & :=\left\{(\psi(t))_{t \in[0, T]} \mathrm{R}^{n}-\text { valued } \mathscr{F}_{T}^{\theta} \vee \mathscr{F}^{B}-\text { adapted process: } E\left[\int_{0}^{T}|\psi(t)|^{2} \mathrm{~d} t\right]<+\infty\right\} .
\end{aligned}
$$

We consider the BSDE with data $(f, \xi)$ :

$$
Y_{t}=\xi+\int_{t}^{T} f\left(s, Y_{s}, Z_{s}\right) \mathrm{d} s-\int_{t}^{T} Z_{s} \mathrm{~d} B_{s}, \quad t \in[0, T] .
$$

Here $f: \Omega \times[0, T] \times R \times R^{d} \longrightarrow R$ is such that, for any $(y, z) \in R \times R^{d}, f(\cdot, y, z)$ is $\mathscr{F}_{T}^{\theta} \vee \mathscr{F}^{B}$-progressively measurable. We make the following assumptions:

(A1) There exists a positive constant $C$ such that for all $\left(t, y_{i}, z_{i}\right) \in[0, T] \times R \times R^{d}, i=1,2$,

$$
\left|f\left(t, y_{1}, z_{1}\right)-f\left(t, y_{2}, z_{2}\right)\right| \leq C\left(\left|y_{1}-y_{2}\right|+\left|z_{1}-z_{2}\right|\right) \text {, a.s. }
$$

(A2) $f(\cdot, 0,0) \in \mathscr{H}^{2}(0, T ; R)$.

Lemma 1. Let assumptions (A1) and (A2) hold; then, for any random variable $\xi \in L^{2}\left(\Omega, \mathscr{F}_{T}, P\right), B S D E$ (12) has a unique solution:

$$
(Y, Z) \in \mathcal{S}^{2}(0, T ; R) \times \mathscr{H}^{2}\left(0, T ; R^{d}\right) .
$$

We give the comparison theorem for solutions of BSDEs.

Lemma 2 (comparison theorem). Let $\xi^{1}, \xi^{2} \in L^{2}\left(\Omega, \mathscr{F}_{T}, P ; R\right)$ and $f^{1}$ and $f^{2}$ satisfy $(A 1)$ and $(A 2)$. We denote by $\left(Y^{1}, Z^{1}\right)$ and $\left(Y^{2}, Z^{2}\right)$ the solutions of BSDEs with data $\left(f^{1}, \xi^{1}\right)$ and $\left(f^{2}, \xi^{2}\right)$, respectively, and we suppose that

(i) $\xi^{1} \leq \xi^{2}, P-a . s$. (ii) $f^{1}\left(t, Y_{t}^{2}, Z_{t}^{2}\right) \leq f^{2}\left(t, Y_{t}^{2}, Z_{t}^{2}\right), d t d P$ - a.e.

Then, we have $Y_{t}^{1} \leq Y_{t}^{2}$, a.s., for all $t \in[0, T]$. Moreover, if $P\left(\xi^{1}<\xi^{2}\right)>0$, then $P\left(Y_{t}^{1}<Y_{t}^{2}\right)>0, t \in[0, T]$, and in particular, $Y_{0}^{1}<Y_{0}^{2}$.

With the notations in the above lemma, we assume that, for some $f:[0, T] \times R \times R^{d} \longrightarrow R$ satisfying (A1) and (A2), the drivers $f_{i}, i=1,2$ have the following form:

$$
\begin{aligned}
& f^{1}(s, y, z)=f(s, y, z)+\varphi_{1}(s), \\
& f^{2}(s, y, z)=f(s, y, z)+\varphi_{2}(s) .
\end{aligned}
$$

Then, we have the following lemma.

Lemma 3. The difference of the solutions $\left(Y^{1}, Z^{1}\right)$ and $\left(Y^{2}, Z^{2}\right)$ of BSDE (12) with the data $\left(\xi^{1}, f^{1}\right)$ and $\left(\xi^{2}, f^{2}\right)$, respectively, satisfies the following estimate:

$$
\begin{aligned}
& \left|Y_{t}^{1}-Y_{t}^{2}\right|+\frac{1}{2} E\left\{\int_{t}^{T} e^{\beta(t-s)}\left[\left|Y_{s}^{1}-Y_{s}^{2}\right|^{2}+\left|Z_{s}^{1}-Z_{s}^{2}\right|^{2}\right] \mathrm{d} s \mid \mathscr{F}_{t}\right\} \\
& \leq E\left\{e^{\beta(T-t)}\left|\xi^{1}-\xi^{2}\right|^{2} \mid \mathscr{F}_{t}\right\} \\
& +E\left\{\int_{t}^{T} e^{\beta(t-s)}\left|\varphi_{1}(s)-\varphi_{2}(s)\right|^{2} \mathrm{~d} s \mid \mathscr{F}_{t}\right\},
\end{aligned}
$$

where $\beta=16\left(1+C^{2}\right)$ and $C$ is the Lipschitz constant in (A1). 
For the proof of the above two lemmas, the readers can refer to $[11,16]$.

We now consider the assumptions on the coefficients $b$ and $\sigma$. The coefficients $b:[0, T] \times R^{n} \times \mathscr{M} \times U \times V \longrightarrow R^{n}$ and $\sigma:[0, T] \times R^{n} \times \mathscr{M} \times U \times V \longrightarrow R^{n \times d}$ are two given functions. The mappings $b$ and $\sigma$ satisfy the following conditions:
(A3)

(i) For every fixed $x \in R^{n}, i \in \mathscr{M}, b(\cdot, x, i, \cdot, \cdot)$ and $\sigma(\cdot, x, i, \cdot, \cdot)$ are continuous with respect to $(t, u, v)$.

(ii) For any $x, x^{\prime} \in R^{n}, i \in \mathscr{M}, u \in U$ and $v \in V$, there exists a positive constant $C$ such that

$$
\left|b(t, x, i, u, v)-b\left(t, x^{\prime}, i, u, v\right)\right|+\left|\sigma(t, x, i, u, v)-\sigma\left(t, x^{\prime}, i, u, v\right)\right| \leq C\left|x-x^{\prime}\right|
$$

From (A3), we can get the global linear growth conditions of $b$ and $\sigma$, i.e., the existence of some $C>0$ such that, for all $t \in[0, T], u \in U, v \in V, x \in R^{n}, i \in \mathscr{M}$,

$$
|b(t, x, i, u, v)|+|\sigma(t, x, i, u, v)| \leq C(1+|x|) .
$$

Suppose the above assumptions hold; for any $u \in U$ and $v \in V$, control system (2) has a unique solution $\left\{X_{s}^{t, x, i ; u, v}, s \in[t, T]\right\}$. And we have the following estimates.

Lemma 4. Under the assumptions of the mappings $b$ and $\sigma$, there exists a positive constant $C$ such that, for any $x, x^{\prime} \in R^{n}, i \in \mathscr{M}$, and $u \in U, v \in V$,

$$
\begin{array}{r}
E\left\{\sup _{s \in[t, T]}\left|X_{s}^{t, x, i ; u, v}\right|^{2} \mid \mathscr{F}_{t}\right\} \leq C\left(1+|x|^{2}\right), \quad P-\text { a.s. } \\
E\left\{\sup _{s \in[t, T]}\left|X_{s}^{t, x, i ; u, v}-X_{s}^{t, x^{\prime}, i ; u, v}\right|^{2} \mid \mathscr{F}_{t}\right\} \leq C\left|x-x^{\prime}\right|^{2}, \quad P-\text { a.s. }
\end{array}
$$

Suppose that the two functions $f:[0, T] \times R^{n} \times R \times R^{d} \times$ $R^{m \times m} \times U \times V \longrightarrow R$ and the terminal cost $\Phi: R^{n} \longrightarrow R$ satisfy the following conditions:

(A4)

(i) For any fixed $(x, y, z, i) \in R^{n} \times R \times R^{d} \times \mathscr{M}$, $f(\cdot, x, y, z, i, \cdot, \cdot)$ is continuous with respect to $(t, u, v)$.

(ii) There exists $C>0$ such that, for all $t \in[0, T]$, $x, x^{\prime} \in R^{n}, y, y^{\prime} \in R, z, z^{\prime} \in R^{d}, i \in M, u \in U$ and $v \in V$,

$$
\begin{aligned}
& \left|f(t, x, y, z, i, u, v)-f\left(t, x^{\prime}, y^{\prime}, z^{\prime}, i, u, v\right)\right| \\
& \quad \leq C\left(\left|x-x^{\prime}\right|+\left(y-y^{\prime}\right)+\left|z-z^{\prime}\right|\right) .
\end{aligned}
$$

(iii) There is a constant $C>0$ such that, for all $x, x^{\prime} \in R^{n}, i \in \mathscr{M}$,

$$
\left|\Phi(x, i)-\Phi\left(x^{\prime}, i\right)\right| \leq C\left|x-x^{\prime}\right| .
$$

Under the above conditions, (3) has a unique solution $\left(Y_{s}^{t, x, i ; u, v}, Z_{s}^{t, x, i ; u, v}\right)_{s \in[t, T]}$. And we have the following estimates.
Lemma 5. For all $t \in[0, T], i \in \mathscr{M}, u \in U$, and $v \in V$, there exists a constant $C>0$ such that,

$$
\begin{array}{r}
\left|Y_{t}^{t, x, i ; u, v}\right| \leq C(1+|x|), \quad P-\text { a.s. } \\
\left|Y_{t}^{t, x, i ; u, v}-Y_{t}^{t, x^{\prime}, i ; u, v}\right| \leq C\left|x-x^{\prime}\right|, \quad P-\text { a.s. }
\end{array}
$$

For the proof of this lemma, the readers can refer to [17].

Now, we introduce the admissible controls and admissible strategies. Let $t_{1}, t_{2}$ be two deterministic times, and $0 \leq t \leq t_{1} \leq t_{2} \leq T$.

Definition 1. An admissible control process $u=\left\{u_{r}, r \in\left[t_{1}, t_{2}\right]\right\}$ (resp., $v=\left\{v_{r}, r \in\left[t_{1}, t_{2}\right]\right\}$ ) for player I (resp., player II) on $\left[t_{1}, t_{2}\right]$ is a process taking values in $U$ (resp., $V$ ), progressively measurable with respect to the filtration $\mathscr{F}$, where $\mathscr{F}=\left\{\mathscr{F}_{r}, r \in\left[t_{1}, t_{2}\right]\right\}$ is the filtration generated by $B$ and $\theta$.

The set of all admissible controls for player I (resp., player II) on time $[t, T]$ is denoted by $U_{t, T}$ (resp., $V_{t, T}$ ).

Definition 2. A nonanticipative strategy for player I on $[t, T]$ is a mapping $\alpha: V \longrightarrow U$ such that, for any $\mathscr{F}_{t}$-stopping time $S$ and any $v, v^{\prime} \in V_{t, T}$, if $v \equiv v^{\prime}$ on $t, S$ (with the notation $t, S=\{(s, \omega) \in[0, T] \times \Omega, t \leq s \leq S(\omega)\})$. In the same way, we define a nonanticipative strategy $\beta: U_{t, T} \longrightarrow V_{t, T}$ for player II on $[t, T]$.

The set of all nonanticipative strategies for player I (resp., player II) on $[t, T]$ is denoted by $\mathscr{A}_{t, T}$ (resp., $\mathscr{B}_{t, T}$ ).

Now we give some properties about the lower and the upper value functions $W$ and $U$. The following lemma was established in [8], and the situation was slightly different. For the proof of this lemma, the readers can refer to [8].

Lemma 6. Under the assumptions (A3) and (A4), for all $(t, x, i) \in[0, T] \times R^{n} \times \mathscr{M}$, the value functions $W(t, x, i)$ and $U(t, x, i)$ are deterministic functions.

From (4), (5), and (22), we get the properties of the lower value function $W$ in the following.

Lemma 7. Under the assumptions (A3) and (A4), for all $(t, x, i) \in[0, T] \times R^{n} \times \mathscr{M}$ and $x, x^{\prime} \in R^{n}$, we have 
(i)

$$
|W(t, x, i)| \leq C(1+|x|) .
$$

(ii) $W(\cdot, x, i)$ is $(1 / 2)$-Hölder continuous with respect to $t:$

$$
\left|W(t, x, i)-W\left(t^{\prime}, x, i\right)\right| \leq C(1+|x|)\left|t-t^{\prime}\right|^{(1 / 2)} .
$$

(iii)

$$
\left|W(t, x, i)-W\left(t, x^{\prime}, i\right)\right| \leq C\left|x-x^{\prime}\right|
$$

The same properties hold true for the function $U$.

\section{Dynamic Programming Principles}

The dynamic programming principle is one of the principal and most commonly used methods to solve the optimal control problem. In this section, we present the dynamic programming principle for a two-player zero-sum SDG with regime switching in the framework of BSDE on a finite time horizon. It will be used in the next section.

We first introduce the backward stochastic semigroup. For given initial state $(t, x, i)$, a positive number $\delta \leq T-t$, for admissible control processes $u$. $\in U_{t, t+\delta}$ and $v . \in V_{t, t+\delta}$, and a real value random variable $\eta \in\left(\Omega, \mathscr{F}_{t+\delta}, P ; R\right)$, we define

$$
G_{t, t+\delta}^{t, x, i u, v}[\eta]:=\bar{Y}_{t}^{t, x, i ; i, v}
$$

where $\left(\bar{Y}_{s}^{t, x ; i ; u, v}, \bar{Z}_{s}^{t, x, i ; u, v}\right)_{s \in[t, t+\delta]}$ is the solution of the following BSDE with terminal time $t+\delta$ :

$$
\begin{aligned}
\bar{Y}_{s}^{t, x ; i ; u, v}= & \eta+\int_{s}^{t+\delta} f\left(r, X_{r}^{t, x, i ; i, \nu, v}, \bar{Y}_{r}^{t, \alpha, i ; u, v}, \bar{Z}_{r}^{t, x, i ; u, v}, \theta_{r}^{t, i}, u_{r}, v_{r}\right) \mathrm{d} r \\
& -\int_{s}^{t+\delta} \bar{Z}_{r}^{t, x, i ; u, v} \mathrm{~d} B_{r}, \quad P-\text { a.s., } s \in[t, t+\delta],
\end{aligned}
$$

and $X^{t, x, i ; u, v}$ is the solution of SDE (2). According to the uniqueness of the solution of the BSDE, we observe that for the solution $Y^{t, x, i ; u, v}$ of BSDE (3), we have

$$
\begin{aligned}
& J(t, x, i ; u, v)=E\left[Y_{t}^{t, x i ; i, v, v} \mid \mathscr{F}_{t}\right]=E\left[G_{t, T}^{t, x ; i ; u, v}\left[\Phi\left(X_{T}^{t, x ; i ; u, v}, \theta_{T}^{t, i}\right)\right] \mid \mathscr{F}_{t}\right] \\
& =E\left[G_{t, t+\delta}^{t, x, i ; u, v}\left[Y_{t+\delta}^{t, x, i ; u, v}\right] \mid \mathscr{F}_{t}\right] \\
& =E\left[G_{t, t+\delta}^{t, x, i, u, v}\left[E\left[Y_{t+\delta}^{t, x, i ; u, v} \mid \mathscr{F}_{t+\delta}\right]\right] \mid \mathscr{F}_{t}\right] \\
& =E\left[G_{t, t+\delta}^{t, x, i, u, v}\left[J\left(t+\delta, X_{t+\delta}^{t, x, i ; u, v}, \theta_{t+\delta}^{t, i} ; u, v\right)\right] \mid \mathscr{F}_{t}\right] .
\end{aligned}
$$

We now introduce the dynamic programming principle for the value functions of SDGs with regime switching.

Proposition 1. Under the assumptions (A3) and (A4), the following dynamic programming principle holds: for all $0<\delta \leq T-t, x \in R^{n}, i \in \mathscr{M}$,

$$
\begin{aligned}
U(t, x, i) & =\underset{\alpha \in \mathscr{A}_{t, t+\delta}}{\operatorname{essup}} \operatorname{essinf} V_{t, t+\delta} E\left[G_{t, t+\delta}^{t, x, i ; \alpha(v), v}\left[U\left(t+\delta, X_{t+\delta}^{t, x, i ; \alpha(v), v}, \theta_{t+\delta}^{t, i}\right)\right] \mid \mathscr{F}_{t}\right], \\
W(t, x, i) & =\underset{\beta \in \mathscr{S}_{t, t+\delta}}{\operatorname{essinf}} \operatorname{esssup} E \in U_{t, t+\delta} E\left[G_{t, t+\delta}^{t, x, i ;, \beta(u)}\left[W\left(t+\delta, X_{t+\delta}^{t, x, i ; u, \beta(u)}, \theta_{t+\delta}^{t, i}\right)\right] \mid \mathscr{F}_{t}\right] .
\end{aligned}
$$

Put

$$
W_{\delta}(t, x, i)=\underset{\beta \in \mathscr{S}_{t, t+\delta}}{\operatorname{essinf}} \operatorname{esssup}_{u \in U_{t, t+\delta}} E\left[G_{t, t+\delta}^{t, x, i ; u, \beta(u)}\left[W\left(t+\delta, X_{t+\delta}^{t, x, i ; u, \beta(u)}, \theta_{t+\delta}^{t, i}\right)\right] \mid \mathscr{F}_{t}\right] .
$$

We proceed with the proof that $W_{\delta}(t, x, i)$ coincides with $W(t, x, i)$ into the following steps.

Step 1. Let $\beta \in \mathscr{B}_{t, T}$ be arbitrarily fixed. Then, given a $u_{2} \in U_{t+\delta, T}$, we define as follows the restriction $\beta_{1}$ of $\beta$ to $U_{t, t+\delta}$ :

$$
\beta_{1}\left(u_{1}\right):=\left.\beta\left(u_{1} \oplus u_{2}\right)\right|_{[t, t+\delta]}, \quad u_{1} \in U_{t, t+\delta},
$$

where $u_{1} \oplus u_{2}:=u_{1} \chi_{[t, t+\delta]}+u_{2} \chi_{(t+\delta, T]}$ extends $u_{1}$ to an element of $U_{t, T}$. Obviously, $\beta_{1} \in \mathscr{B}_{t, t+\delta}$. And, from the nonanticipative property of $\beta$ we deduce that $\beta_{1}$ is independent of the special choice of $u_{2} \in U_{t+\delta, T}$. Thus, from the definition of $W_{\delta}(t, x, i)$,

$$
W_{\delta}(t, x, i) \leq \operatorname{esssup}_{u_{1} \in U_{t, t+\delta}} E\left[G_{t, t+\delta}^{t, x, i ; u_{1}, \beta_{1}}\left(u_{1}\right)\left[W\left(t+\delta, X_{t+\delta}^{t, x, i ; u_{1}, \beta_{1}\left(u_{1}\right)}, \theta_{t+\delta}^{t, i}\right)\right] \mid \mathscr{F}_{t}\right],
$$


and we use the notation $\mathscr{I}_{\delta}(t, x, i ; u, v)$ $:=E\left[G_{t, t+\delta}^{t, x, i ; u, v}\left[W\left(t+\delta, X_{t+\delta}^{t, x, i ; u, v}, \theta_{t+\delta}^{t, i}\right)\right] \mid \mathscr{F}_{t}\right]$ for some sequences $\left\{u_{l}^{1}, l \geq 1\right\} \subset U_{t, t+\delta}$ such that,

$$
\mathscr{I}_{\delta}\left(t, x, i ; \beta_{1}\right):=\operatorname{esssup}_{u_{1} \in U_{t, t+\delta}} \mathscr{I}_{\delta}\left(t, x, i ; u_{1}, \beta_{1}\left(u_{1}\right)\right)=\sup _{l \geq 1} \mathscr{I}_{\delta}\left(t, x, i ; u_{l}^{1}, \beta_{1}\left(u_{l}^{1}\right)\right), \quad P-\text { a.s. }
$$

Let $\varepsilon>0$ and set $\widetilde{\Gamma}_{l}:=\left\{\mathscr{I}_{\delta}\left(t, x, i ; \beta_{1}\right) \leq \mathscr{I}_{\delta}(t, x, i\right.$; $\left.\left.u_{l}^{1}, \beta_{1}\left(u_{l}^{1}\right)\right)+\varepsilon\right\} \in \mathscr{F}_{t}, l \geq 1$. Construct $\Gamma_{1}:=\widetilde{\Gamma}_{1}, \quad \Gamma_{l}:=$ $\widetilde{\Gamma}_{l},\left(\cup_{k=1}^{l-1} \widetilde{\Gamma}_{k}\right) \in \mathscr{F}_{t}, l \geq 2$. Certainly, $\left\{\Gamma_{l}\right\}_{l \geq 1}$ forms an $(\Omega$, $\left.\mathscr{F}_{t}\right)$-partition, and $u_{1}^{\varepsilon}:=\sum_{l \geq 1} \chi_{\Gamma_{l}} u_{l}^{1} \in U_{t, t+\delta}$. Moreover, from the nonanticipativity of $\beta_{1}$, we have $\beta_{1}\left(u_{1}^{\varepsilon}\right)=$ $\sum_{l \geq 1} \chi_{\Gamma_{l}} \beta_{1}\left(u_{l}^{1}\right)$. According to the existence and uniqueness of the BSDEs, it follows that

$$
\mathscr{I}_{\delta}\left(t, x, i ; u_{1}^{\varepsilon}, \beta_{1}\left(u_{1}^{\varepsilon}\right)\right)=\sum_{l \geq 1} \chi_{\Gamma_{l}} \mathscr{I}_{\delta}\left(t, x, i ; u_{l}^{1}, \beta_{1}\left(u_{l}^{1}\right)\right), \quad P \text { - a.s. }
$$

for $\beta_{1} \in \mathscr{B}_{t, t+\delta}$. Hence,

$$
\begin{aligned}
W_{\delta} & (t, x, i) \\
& \leq \mathscr{I}_{\delta}\left(t, x, i ; \beta_{1}\right) \\
& \leq \sum_{l \geq 1} \chi_{\Gamma_{l}} \mathscr{I}_{\delta}\left(t, x, i ; u_{l}^{1}, \beta_{1}\left(u_{l}^{1}\right)\right)+\varepsilon \\
& =\mathscr{I}_{\delta}\left(t, x, i ; u_{1}^{\varepsilon}, \beta_{1}\left(u_{1}^{\varepsilon}\right)\right)+\varepsilon \\
& =E\left[G_{t, t+\delta}^{t, x, i ; u_{1}^{\varepsilon}, \beta_{1}}\left(u_{1}^{\varepsilon}\right)\left[W\left(t+\delta, X_{t+\delta}^{t, x, i ; u_{1}^{\varepsilon}, \beta_{1}\left(u_{1}^{\varepsilon}\right)}, \theta_{t+\delta}^{t, i}\right)\right] \mid \mathscr{F}_{t}\right]+\varepsilon, \quad P-\text { a.s. }
\end{aligned}
$$

We now focus on the interval $[t+\delta, T]$. Because $W(t+\delta, y, j) \leq \underset{u_{2} \in U_{t+\delta, T}}{\operatorname{esssup}} J\left(t+\delta, y, j ; u_{2}, \beta_{2}\left(u_{2}\right)\right), \quad P-$ a.s. $\beta_{1}(\cdot):=\beta\left(\cdot \oplus u_{2}\right) \in \mathscr{B}_{t, t+\delta}$ does not depend on

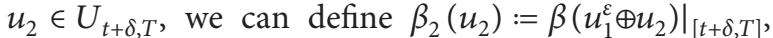
for any $u_{2} \in U_{t+\delta, T}$. From $\beta \in \mathscr{B}_{t, T}$, we know that $\beta_{2}: U_{t+\delta, T} \longrightarrow V_{t+\delta, T}$ belongs to $\mathscr{B}_{t+\delta, T}$. Thus, from the definition of $W(t+\delta, y, j)$, for any $(y, j) \in R^{n} \times \mathscr{M}$,

From Lemmas 5 and 7, there exists a constant $C \in R$ such that, for any $u_{2} \in U_{t+\delta, T}, y, y^{\prime} \in R^{n}$,

(i) $\left|W(t+\delta, y, j)-W\left(t+\delta, y^{\prime}, j\right)\right| \leq C\left|y-y^{\prime}\right|$,

(ii) $\left|J\left(t+\delta, y, j ; u_{2}, \beta_{2}\left(u_{2}\right)\right)-J\left(t+\delta, y^{\prime}, j ; u_{2}, \beta_{2}\left(u_{2}\right)\right)\right| \leq C\left|y-y^{\prime}\right|, \quad P-$ a.s.

We can show by approximating $X_{t+\delta}^{t, x, i ; u_{1}^{\varepsilon}, \beta_{1}\left(u_{1}^{\varepsilon}\right)}$ that

$$
\begin{aligned}
& W\left(t+\delta, X_{t+\delta}^{t, x, i ; u_{1}^{\varepsilon}, \beta_{1}\left(u_{1}^{\varepsilon}\right)}, \theta_{t+\delta}^{t, i}\right) \\
& \quad \leq \operatorname{esssup}_{u_{2} \in U_{t+\delta, T}} J\left(t+\delta, X_{t+\delta}^{t, x, i ; u_{1}^{\varepsilon}, \beta_{1}\left(u_{1}^{\varepsilon}\right)}, \theta_{t+\delta}^{t, i} ; u_{2}, \beta_{2}\left(u_{2}\right)\right), \quad P-\text { a.s. }
\end{aligned}
$$

To estimate the right side of the latter inequality, we note that there exists some sequence $\left\{u_{k}^{2}, k \geq 1\right\} \subset U_{t+\delta, T}$ such that

$$
\begin{aligned}
& \operatorname{esssup}_{u_{2} \in U_{t+\delta, T}} J\left(t+\delta, X_{t+\delta}^{t, x, i ; u_{1}^{\varepsilon}, \beta_{1}\left(u_{1}^{\varepsilon}\right)}, \theta_{t+\delta}^{t, i} ; u_{2}, \beta_{2}\left(u_{2}\right)\right) \\
& \quad=\sup _{k \geq 1} J\left(t+\delta, X_{t+\delta}^{t, x, i ; u_{1}^{\varepsilon}, \beta_{1}\left(u_{1}^{\varepsilon}\right)}, \theta_{t+\delta}^{t, i}, u_{k}^{2}, \beta_{2}\left(u_{k}^{2}\right)\right), \quad P-\text { a.s. }
\end{aligned}
$$

Let $\varepsilon>0$ and set $\widetilde{\triangle}_{k}:=\left\{\operatorname{esssup}_{u_{2} \in U_{t+\delta T}} J(t+\delta\right.$, $\left.X_{t+\delta}^{t, x, i ; u_{1}^{\varepsilon}, \beta_{1}\left(u_{1}^{\varepsilon}\right)}, \theta_{t+\delta}^{t, i} ; u_{2}, \beta_{2} \quad\left(u_{2}\right)\right) \leq J\left(t+\delta, X_{t+\delta}^{t, x, i ; u_{1}^{\varepsilon}, \beta_{1}\left(u_{1}^{\varepsilon}\right)}\right.$, $\left.\left.\theta_{t+\delta}^{t, i} ; u_{k}^{2}, \beta_{2}\left(u_{k}^{2}\right)\right)+\varepsilon\right\} \in \mathscr{F}_{t+\delta}, k \geq 1$. Construct $\Delta_{1}:=\widetilde{\Delta}_{1}$, $\Delta_{k}:=\widetilde{\Delta}_{k},\left(\cup_{j=1}^{k-1} \widetilde{\Delta}_{j}\right) \in \mathscr{F}_{t+\delta}, k \geq 2$. Certainly, $\left\{\Delta_{k}\right\}_{k \geq 1}$ 
forms an $\left(\Omega, \mathscr{F}_{t+\delta}\right)$-partition; moreover, $u_{2}^{\varepsilon}:=\sum_{k \geq 1} \chi_{\triangle} u_{k}^{2} \in U_{t+\delta, T}$. Therefore, from the nonanticipativity of $\beta_{2}$, we have $\beta_{2}\left(u_{2}^{\varepsilon}\right)=\sum_{k \geq 1} \chi_{\triangle_{k}} \beta_{2}\left(u_{k}^{2}\right)$, and from the definition of $\beta_{1}, \beta_{2}$, we know that $\beta\left(u_{1}^{\varepsilon} \oplus u_{2}^{\varepsilon}\right)=\beta_{1}\left(u_{1}^{\varepsilon}\right) \oplus \beta_{2}\left(u_{2}^{\varepsilon}\right)$. According the existence and uniqueness of our BSDE, it follows that

$$
\begin{aligned}
& J\left(t+\delta, X_{t+\delta}^{t, x, i ; u_{1}^{\varepsilon}, \beta_{1}\left(u_{1}^{\varepsilon}\right)}, \theta_{t+\delta}^{t, i} ; u_{2}^{\varepsilon}, \beta_{2}\left(u_{2}^{\varepsilon}\right)\right)=E\left[Y_{t+\delta}^{t+\delta, X_{t+\delta}^{t, x ; i} u_{1}^{\varepsilon}, \beta_{1}\left(u_{1}^{\varepsilon}\right)}, \theta_{t+\delta}^{t, i} ; u_{2}^{\varepsilon}, \beta_{2}\left(u_{2}^{\varepsilon}\right) \mid \mathscr{F}_{t+\delta}\right] \\
& =E\left[\sum_{k \geq 1} \chi_{\Delta_{k}} Y_{t+\delta}^{t+\delta, X_{t+\delta}^{t, x ; i}, u_{1}^{\varepsilon}, \beta_{1}\left(u_{1}^{\varepsilon}\right)}, \theta_{t+\delta}^{t, i} ; u_{k}^{\varepsilon}, \beta_{2}\left(u_{k}^{\varepsilon}\right) \mid \mathscr{F}_{t+\delta}\right] \\
& =\sum_{k \geq 1} \chi_{\Delta_{k}} J\left(t+\delta, X_{t+\delta}^{t, x, i ; u_{1}^{\varepsilon}, \beta_{1}\left(u_{1}^{\varepsilon}\right)}, \theta_{t+\delta}^{t, i} ; u_{k}^{\varepsilon}, \beta_{2}\left(u_{k}^{\varepsilon}\right)\right), \quad P-\text { a.s. }
\end{aligned}
$$

Therefore, where $u^{\varepsilon}:=u_{1}^{\varepsilon} \oplus u_{2}^{\varepsilon} \in U_{t, T}$. From (35) and (41),

$$
\begin{aligned}
& W\left(t+\delta, X_{t+\delta}^{t, x, i ; u_{1}^{\varepsilon}, \beta_{1}\left(u_{1}^{\varepsilon}\right)}, \theta_{t+\delta}^{t, i}\right) \\
& \leq \operatorname{esssup}_{u_{2} \in U_{t+\delta, T}}\left(t+\delta, X_{t+\delta}^{t, x, i ; u_{1}^{\varepsilon}, \beta_{1}\left(u_{1}^{\varepsilon}\right)}, \theta_{t+\delta}^{t, i} ; u_{2}, \beta_{2}\left(u_{2}\right)\right) \\
& \leq E\left[\sum_{k \geq 1} \chi_{\Delta_{k}} Y_{t+\delta}^{t, x, i ; u_{1}^{\varepsilon} \oplus u_{k}^{2}, \beta\left(u_{1}^{\varepsilon} \oplus u_{k}^{2}\right)} \mid \mathscr{F}_{t+\delta}\right]+\varepsilon \\
& \quad=E\left[Y_{t+\delta}^{t, x, i ; u_{1}^{\varepsilon} \oplus u_{2}^{\varepsilon}, \beta\left(u_{1}^{\varepsilon} \oplus u_{2}^{\varepsilon}\right)} \mid \mathscr{F}_{t+\delta}\right]+\varepsilon \\
& \quad=E\left[Y_{t+\delta}^{t, x, i ; u^{\varepsilon}, \beta\left(u^{\varepsilon}\right)} \mid \mathscr{F}_{t+\delta}\right]+\varepsilon, \quad P-\text { a.s., }
\end{aligned}
$$

$$
\begin{aligned}
& \begin{aligned}
W_{\delta}(t, x, i) & \leq E\left[G_{t, t+\delta}^{t, x, i ; u_{1}^{\varepsilon}, \beta_{1}\left(u_{1}^{\varepsilon}\right)}\left[E\left[Y_{t+\delta}^{t, x, i ; u^{\varepsilon}, \beta\left(u^{\varepsilon}\right)} \mid \mathscr{F}_{t+\delta}\right]+\varepsilon\right] \mid \mathscr{F}_{t}\right] \\
& \leq E\left[G_{t, t+\delta}^{t, x, i ; u_{1}^{\varepsilon}, \beta_{1}\left(u_{1}^{\varepsilon}\right)}\left[E\left[Y_{t+\delta}^{t, x, i ; u^{\varepsilon}, \beta\left(u^{\varepsilon}\right)} \mid \mathscr{F}_{t+\delta}\right]\right] \mid \mathscr{F}_{t}\right]+(C+1) \mathcal{\varepsilon}
\end{aligned} \\
& =E\left[G_{t, t+\delta}^{t, x, i ; u^{\varepsilon}, \beta\left(u^{\varepsilon}\right)}\left[E\left[Y_{t+\delta}^{t, x, i ; u^{\varepsilon}, \beta\left(u^{\varepsilon}\right)} \mid \mathscr{F}_{t+\delta}\right]\right] \mid \mathscr{F}_{t}\right]+(C+1) \varepsilon \\
& =E\left[G_{t, t+\delta}^{t, x, i ; u^{\varepsilon}, \beta\left(u^{\varepsilon}\right)}\left[Y_{t+\delta}^{t, x, i ; u^{\varepsilon}, \beta\left(u^{\varepsilon}\right)}\right] \mid \mathscr{F}_{t}\right]+(C+1) \mathcal{E} \\
& =E\left[Y_{t}^{t, x, i ; u^{\varepsilon}, \beta\left(u^{\varepsilon}\right)} \mid \mathscr{F}_{t}\right]+(C+1) \varepsilon \\
& \leq \underset{u \in U_{t, T}}{\operatorname{esssup}} E\left[Y_{t}^{t, x, i ; u, \beta(u)} \mid \mathscr{F}_{t}\right]+(C+1) \varepsilon, \quad P-\text { a.s. }
\end{aligned}
$$

Since $\beta \in \mathscr{B}_{t, T}$ has been arbitrarily chosen, we have (42) for all $\beta \in \mathscr{B}_{t, T}$. Thus,

$$
\begin{aligned}
W_{\delta}(t, x, i) & \leq \underset{\beta \in \mathscr{B}_{t, T}}{\operatorname{essinf}} \operatorname{esssup} E\left[Y_{t}^{t, x, i ; u, \beta(u)} \mid \mathscr{F}_{t}\right]+(C+1) \mathcal{\varepsilon} \\
& =W(t, x, i)+(C+1) \varepsilon .
\end{aligned}
$$

Then, letting $\varepsilon \longrightarrow 0$, we get $W_{\delta}(t, x, i) \leq W(t, x, i)$. Step 2. We now deal with the other case: $W(t, x, i) \leq W_{\delta}(t, x, i)$. From the definition of $W_{\delta}(t, x, i)$, we have

$$
\begin{aligned}
W_{\delta}(t, x, i) & =\underset{\beta_{1} \in \mathscr{B}_{t, t+\delta}}{\operatorname{essinf}} \operatorname{essup} \in U_{t, t+\delta} E\left[G_{t, t+\delta}^{t, x, i ; u_{1}, \beta_{1}\left(u_{1}\right)}\left[W\left(t+\delta, X_{t+\delta}^{t, x, i ; u_{1}, \beta_{1}\left(u_{1}\right)}, \theta_{t+\delta}^{t, i}\right)\right] \mid \mathscr{F}_{t}\right] \\
& =\underset{\beta_{1} \in \mathscr{B}_{t, t+\delta}}{\operatorname{essinf}} \mathscr{I}_{\delta}\left(t, x, i ; \beta_{1}\right),
\end{aligned}
$$


for some $\left\{\beta_{l}^{1}, l \geq 1\right\} \subset \mathscr{B}_{t, t+\delta}$ such that,

$$
W_{\delta}(t, x, i)=\inf _{l \geq 1} \mathscr{I}_{\delta}\left(t, x, i ; \beta_{l}^{1}\right), \quad P-\text { a.s. }
$$

For any $\varepsilon>0$, we put $\tilde{\Lambda}_{l}:=\left\{\mathscr{F}_{\delta}\left(t, x, i ; \beta_{l}^{1}\right)-\varepsilon \leq W_{\delta}\right.$ $(t, x, i)\} \in \mathscr{F}_{t}, l \geq 1, \quad \Lambda:=\widetilde{\Lambda}_{1}, \quad$ and $\Lambda_{l}:=\widetilde{\Lambda}_{l},\left(\cup_{k=1}^{l-1} \widetilde{\Lambda}_{k}\right) \in$ $\mathscr{F}_{t}, l \geq 2$. Certainly, $\left\{\Lambda_{l}\right\}_{l \geq 1}$ forms an $\left(\Omega, \mathscr{F}_{t}\right)$-partition; moreover, $\beta_{1}^{\varepsilon}:=\sum_{l \geq 1} \chi_{\Lambda_{l}} \beta_{l}^{1} \in \mathscr{B}_{t, t+\delta}$. According the existence and uniqueness of our BSDE, we conclude that

$$
\mathscr{I}_{\delta}\left(t, x, i ; u_{1}, \beta_{1}^{\varepsilon}\left(u_{1}\right)\right)=\sum_{l \geq 1} \chi_{\Lambda_{l}} \mathscr{I}_{\delta}\left(t, x, i ; u_{1}, \beta_{l}^{1}\left(u_{1}\right)\right), \quad P-\text { a.s },
$$

for all $u_{1} \in U_{t, t+\delta}$. Next,

$$
\begin{aligned}
& W_{\delta}(t, x, i) \\
& \quad \geq \sum_{l \geq 1} \chi_{\Lambda_{l}} \mathscr{I}_{\delta}\left(t, x, i ; \beta_{l}^{1}\right)-\varepsilon \\
& \quad \geq \sum_{l \geq 1} \chi_{\Lambda_{l}} \mathscr{I}_{\delta}\left(t, x, i ; u_{1}, \beta_{l}^{1}\left(u_{1}\right)\right)-\varepsilon \\
& \quad=\mathscr{I}_{\delta}\left(t, x, i ; u_{1}, \beta_{1}^{\varepsilon}\left(u_{1}\right)\right)-\varepsilon
\end{aligned}
$$$$
=E\left[G_{t, t+\delta}^{t, x, i ; u_{1}, \beta_{1}^{\varepsilon}\left(u_{1}\right)}\left[W\left(t+\delta, X_{t+\delta}^{t, x, i ; u_{1}, \beta_{1}^{\varepsilon}\left(u_{1}\right)}, \theta_{t+\delta}^{t, i}\right)\right] \mid \mathscr{F}_{t}\right]-\varepsilon, \quad P-\text { a.s, }
$$

for all $u_{1} \in U_{t, t+\delta}$.

We now focus on the interval $[t+\delta, T]$. From the definition of $W(t+\delta, y, j)$, we deduce that, for any $(y, j) \in R^{n} \times \mathscr{M}$, there exists $\beta_{y, j}^{\varepsilon} \in \mathscr{B}_{t+\delta, T}$ such that

$$
W(t+\delta, y, j) \geq \underset{u_{2} \in U_{t+\delta, T}}{\operatorname{esssup}} J\left(t+\delta, y, j ; u_{2}, \beta_{y, j}^{\varepsilon}\left(u_{2}\right)\right)-\varepsilon, \quad P-\text { a.s. }
$$

Now consider a decomposition of $R^{n}$, namely, $\sum_{l \geq 1} O_{l}=$ $R^{n}$ such that $\operatorname{diam}\left(O_{l}\right) \leq \varepsilon$, for each $l \geq 1$. Take any $\left(y_{l}, j\right) \in O_{l} \times \mathscr{M}, l \geq 1$ fixed and define

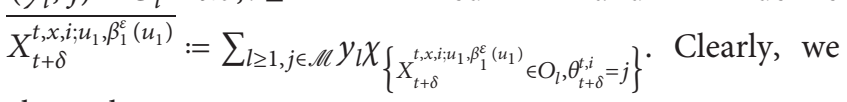
always have

$$
\left|X_{t+\delta}^{t, x, i ; u_{1}, \beta_{1}^{\varepsilon}\left(u_{1}\right)}-\overline{X_{t+\delta}^{t, x i ; u_{1}, \beta_{1}^{\varepsilon}\left(u_{1}\right)}}\right| \leq \varepsilon,
$$

everywhere on $\Omega$, for each $u_{1} \in U_{t, t+\delta}$. Moreover, for every $y_{l}$ and $j$, there exists some $\beta_{y_{b}, j}^{\varepsilon} \in \mathscr{B}_{t+\delta, T}$ such that (48) holds, and clearly,

$$
\beta_{u_{1}}^{\varepsilon}:=\sum_{l \geq 1, j \in \mathscr{M}} \chi_{\left\{X_{t+\delta}^{t, x ; i, u_{1}, \beta_{1}^{\varepsilon}\left(u_{1}\right)} \in \mathrm{O}_{l}, \theta_{t+\delta}^{t, i}=j\right\}} \beta_{y_{l}, j}^{\varepsilon} \in \mathscr{B}_{t+\delta, T} .
$$

Now we can define the new strategy $\beta^{\varepsilon}(u):=\beta_{1}^{\varepsilon}\left(u_{1}\right) \oplus \beta_{u_{1}}^{\varepsilon}\left(u_{2}\right), u \in U_{t, T}$, where $u_{1}=\left.u\right|_{[t, t+\delta]}, u_{2}=$ $\left.u\right|_{(t+\delta, T]}$. Obviously, $\beta^{\varepsilon}: U_{t, T} \longrightarrow V_{t, T}$.

Next we shall show that $\beta^{\varepsilon}$ is nonanticipating: indeed, let $\mathcal{S}: \Omega \longrightarrow[t, T]$ be an $\mathscr{F}$-stopping time and $u, u^{\prime} \in U_{t, T}$ be such that $u \equiv u^{\prime}$ on $t, \mathcal{S}$. Decomposing $u, u^{\prime}$ into $u_{1}, u_{1}^{\prime} \in U_{t, t+\delta}, \quad u_{2}, u_{2}^{\prime} \in U_{t+\delta, T}$ such that $u=u_{1} \oplus u_{2}$ and $u^{\prime}=u_{1}^{\prime} \oplus u_{2}^{\prime}$. We have $u_{1} \equiv u_{1}^{\prime}$ on $t, \delta \wedge(t+\delta)$ since $\beta_{1}^{\varepsilon}\left(u_{1}\right) \equiv \beta_{1}^{\varepsilon}\left(u_{1}^{\prime}\right)$ on $t, \delta \wedge(t+\delta)$. On the other hand, $u_{2} \equiv u_{2}^{\prime}$ on $\rrbracket t+\delta, \delta \wedge(t+\delta) \rrbracket(\subset(t+\delta, T] \times\{\delta>t+\delta\})$, and on $\mathcal{S}>t+\delta$, we have $X_{t+\delta}^{t, x, i ; u_{1}, \beta_{1}^{\varepsilon}\left(u_{1}\right)}=X_{t+\delta}^{t, x, i ; u_{1}^{\prime} \beta_{1}^{\varepsilon}\left(u_{1}^{\prime}\right)}$. Thus, from the definition, $\beta_{u_{1}}^{\varepsilon}=\beta_{u_{1}^{\prime}}^{\varepsilon}$ on $\{\mathcal{S}>t+\delta\}$ and $\beta_{u_{1}}^{\varepsilon}\left(u_{2}\right) \equiv \beta_{u_{1}^{\prime}}^{\varepsilon}\left(u_{2}^{\prime}\right)$ on $\quad \rrbracket t+\delta, \delta \vee(t+\delta) \rrbracket$. This yields $\quad \beta^{\varepsilon}(u)=$ $\beta_{1}^{\varepsilon}\left(u_{1}\right) \oplus \beta_{u_{1}}^{\varepsilon}\left(u_{2}\right) \equiv \beta_{1}^{\varepsilon}\left(u_{1}^{\prime}\right) \oplus \beta_{u_{1}^{\prime}}^{\varepsilon}\left(u_{2}^{\prime}\right)=\beta^{\varepsilon}\left(u^{\prime}\right)$ on $t, \mathcal{S}$, from which it follows that $\beta^{\varepsilon} \in \mathscr{B}_{t, T}$.

Fix $u \in U_{t, T}$ arbitrarily and decompose into $u_{1}=u$

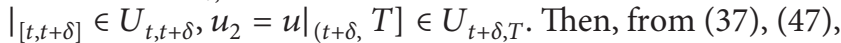
(i), and (49), we have

$$
\begin{aligned}
& W_{\delta}(t, x, i) \\
& \quad \geq E\left[G_{t, t+\delta}^{t, x, i ; u_{1}, \beta_{1}^{\varepsilon}\left(u_{1}\right)}\left[W\left(t+\delta, X_{t+\delta}^{t, x, i ; u_{1}, \beta_{1}^{\varepsilon}\left(u_{1}\right)}, \theta_{t+\delta}^{t, i}\right)\right] \mid \mathscr{F}_{t}\right]-\varepsilon \\
& \quad \geq E\left[G_{t, t+\delta}^{t, x, i ; u_{1}, \beta_{1}^{\varepsilon}\left(u_{1}\right)}\left[W\left(t+\delta, \overline{X_{t+\delta}^{t, x, i ; u_{1}, \beta_{1}^{\varepsilon}\left(u_{1}\right)}}, \theta_{t+\delta}^{t, i}\right)-C \varepsilon\right] \mid \mathscr{F}_{t}\right]-\varepsilon \\
& \quad \geq E\left[G_{t, t+\delta}^{t, x, i ; u_{1}, \beta_{1}^{\varepsilon}\left(u_{1}\right)}\left[W\left(t+\delta, \overline{X_{t+\delta}^{t, x, i ; u_{1}, \beta_{1}^{\varepsilon}\left(u_{1}\right)}}, \theta_{t+\delta}^{t, i}\right)\right] \mid \mathscr{F}_{t}\right]-C \varepsilon \\
& \quad=E\left[G_{t, t+\delta}^{t, x, i ; u_{1}, \beta_{1}^{\varepsilon}\left(u_{1}\right)}\left[\sum_{l \geq 1, j \in \mathscr{M}} \chi_{\left\{X_{t+\delta}^{t, x ; u_{1}, \beta_{1}^{\varepsilon}\left(u_{1}\right)} \in O_{l}, \theta_{t+\delta}^{t, i}=j\right\}} W\left(t+\delta, y_{l}, j\right)\right] \mid \mathscr{F}_{t}\right]-C \varepsilon .
\end{aligned}
$$

From (37), (48), (ii), and (49), it follows that 


$$
\begin{aligned}
& W_{\delta}(t, x, i) \\
& \geq E\left[G_{t, t+\delta}^{t, x, i ; u_{1}, \beta_{1}^{\varepsilon}\left(u_{1}\right)}\left[\sum_{l \geq 1, j \in \mathscr{M}} \chi_{\left\{X_{t+\delta}^{t, x, i ; u_{1}, \beta_{1}^{\varepsilon}\left(u_{1}\right)} \in O_{l}, \theta_{t+\delta}^{t, i}=j\right\}} J\left(t+\delta, y_{l}, j ; u_{2}, \beta_{y_{l}}^{\varepsilon}\left(u_{2}\right)\right)-\varepsilon\right] \mid \mathscr{F}_{t}\right]-C \varepsilon \\
& \geq E\left[G _ { t , t + \delta } ^ { t , x , i ; u _ { 1 } , \beta _ { 1 } ^ { \varepsilon } ( u _ { 1 } ) } \left[\sum_{l \geq 1, j \in \mathscr{M}} \chi_{\left\{X_{t+\delta}^{t, x, i ; u_{1}, \beta_{1}^{\varepsilon}\left(u_{1}\right)} \in \mathrm{O}_{l}, \theta_{t+\delta}^{t, i}=j\right\}}\right.\right. \\
& \left.\left.J\left(t+\delta, y_{l}, j ; u_{2}, \beta_{y_{l}}^{\varepsilon}\left(u_{2}\right)\right)\right] \mid \mathscr{F}_{t}\right]-C \varepsilon \\
& =E\left[G_{t, t+\delta}^{t, x, i ; u_{1}, \beta_{1}^{\varepsilon}\left(u_{1}\right)}\left[J\left(t+\delta, \overline{X_{t+\delta}^{t, x, i ; u_{1}, \beta_{1}^{\varepsilon}\left(u_{1}\right)}}, \theta_{t+\delta}^{t, i} ; u_{2}, \beta_{u_{1}}^{\varepsilon}\left(u_{2}\right)\right)\right] \mid \mathscr{F}_{t}\right]-C \varepsilon \\
& \geq E\left[G_{t, t+\delta}^{t, x, i ; u_{1}, \beta_{1}^{\varepsilon}\left(u_{1}\right)}\left[J\left(t+\delta, X_{t+\delta}^{t, x, i ; u_{1}, \beta_{1}^{\varepsilon}\left(u_{1}\right)}, \theta_{t+\delta}^{t, i} ; u_{2}, \beta_{u_{1}}^{\varepsilon}\left(u_{2}\right)\right)-C \varepsilon\right] \mid \mathscr{F}_{t}\right]-C \varepsilon \\
& \geq E\left[G_{t, t+\delta}^{t, x, i ; u_{1}, \beta_{1}^{\varepsilon}\left(u_{1}\right)}\left[J\left(t+\delta, X_{t+\delta}^{t, x, i ; u_{1}, \beta_{1}^{\varepsilon}\left(u_{1}\right)}, \theta_{t+\delta}^{t, i} ; u_{2}, \beta_{u_{1}}^{\varepsilon}\left(u_{2}\right)\right)\right] \mid \mathscr{F}_{t}\right]-C \varepsilon \\
& =E\left[G_{t, t+\delta}^{t, x, i ; u, \beta^{\varepsilon}(u)}\left[E\left[Y_{t+\delta}^{t, x, i ; u, \beta^{\varepsilon}(u)} \mid \mathscr{F}_{t+\delta}\right]\right] \mid \mathscr{F}_{t}\right]-C \varepsilon \\
& =E\left[G_{t, t+\delta}^{t, x, i ; u, \beta^{\varepsilon}(u)}\left[Y_{t+\delta}^{t, x, i ; u, \beta^{\varepsilon}(u)}\right] \mid \mathscr{F}_{t}\right]-C \varepsilon \\
& =E\left[Y_{t}^{t, x, i ; u, \beta^{\varepsilon}(u)} \mid \mathscr{F}_{t}\right]-C \mathcal{E}, \quad P-\text { a.s., }
\end{aligned}
$$

for any $u \in U_{t, T}$. Therefore, we obtain

$$
\begin{aligned}
W_{\delta}(t, x, i) & \geq \underset{u \in U_{t, T}}{\operatorname{esssup}} J\left(t, x, i ; u, \beta^{\varepsilon}(u)\right)-C \varepsilon \\
& \geq \underset{\beta \in \mathscr{B}_{t, T}}{\operatorname{essinf}} \underset{u \in U_{t, T}}{\operatorname{esssup}} J(t, x, i ; u, \beta(u))-C \varepsilon \\
& =W(t, x, i)-C \varepsilon, \quad P-\text { a.s. }
\end{aligned}
$$

Let $\varepsilon \longrightarrow 0$, and we have $W_{\delta}(t, x, i) \geq W(t, x, i)$.

The proof is complete.

\section{Viscosity Solution of Isaacs' Equation: Existence and Uniqueness Theorem}

In this section, based on the dynamic programming principle, we want to prove that the lower value function $W(t, x, i)$ introduced by (5) is the viscosity solution of (7), while the upper value function $U(t, x, i)$ defined by $(6)$ is the viscosity solution of (8). Moreover, if Isaacs' condition holds, i.e., $H^{-}=H^{+}$, then (7) and (8) coincide, and the uniqueness of viscosity solution implies that $W=U$, that is, the game admits a value.

We first recall the definition of a viscosity solution of (7). The one for (8) can be defined in a similar way

Definition 3. A continuous $W \in\left(C\left([0, T] \times R^{n}\right)\right)^{m}$ is said to be a viscosity subsolution (resp., supersolution) of (7), if $W(T, x, i) \leq \Phi(x, i) \quad$ (resp., $W(T, x, i) \geq \Phi(x, i))$, for all $(x, i) \in R^{n} \times \mathscr{M}$ and if for all functions $\phi \in C_{l, b}^{3}\left([0, T] \times R^{n}\right)$ and $(t, x, i) \in[0, T) \times R^{n} \times \mathscr{M}$ such that $W(\cdot, \cdot)-\phi(\cdot)$ attains its local maximum (resp., minimum) value zero at $(t, x)$, it has

$$
\frac{\partial \phi}{\partial t}(t, x)+H^{-}\left(t, x, i, \phi, D \phi, D^{2} \phi\right)+\sum_{k \neq i} q_{i k}[W(t, x, k)-W(t, x, i)] \leq 0,
$$

(resp., $\geq 0$ ), 
where $W$ is called a viscosity solution if it is both a viscosity subsolution and a viscosity supersolution.

Remark 1. $C_{l, b}^{3}\left([0, T] \times R^{n}\right)$ denotes the set of the realvalued functions that are continuously differentiable up to the third order and whose derivatives of order from 1 to 3 are bounded.

In the following, we prove that the lower value function $W$ is a viscosity solution of (7). We only focus on the lower value function $W$, and the results hold for the upper value function $U$ in a similar procedure.

Theorem 1. Under the assumptions (A3) and (A4), the lower value function $W(t, x, i)$ is a viscosity solution of $(7)$.

First we prove some auxiliary lemmas. To abbreviate notations, for some arbitrarily chosen but fixed $\varphi(\cdot, \cdot) \in C_{l, b}^{3}\left([0, T] \times R^{n}\right)$,

$$
\begin{aligned}
F(s, x, y, z, i, u, v)= & \frac{\partial \varphi}{\partial s}(s, x)+\frac{1}{2} \operatorname{Tr}\left(\sigma \sigma^{T}(s, x, i, u, v) D^{2} \varphi\right)+D \varphi \cdot b(s, x, i, u, v) \\
& +f(s, x, y+\varphi(s, x), z+\varphi(s, x) \cdot \sigma(s, x, i, u, v), i, u, v) \\
& +\sum_{k \neq i} q_{i k}[W(s, x, k)-W(s, x, i)],
\end{aligned}
$$

where $(s, x, y, z, i, u, v) \in[0, T] \times R^{n} \times R \times R^{d} \times \mathscr{M} \times U \times V$ and $\sum_{k \neq i} q_{i k}<1$, and we consider the following BSDE defined on the interval $[t, t+\delta](0<\delta \leq T-t)$ :

$$
\left\{\begin{array}{l}
-\mathrm{d} Y_{s}^{1, u, v}=F\left(s, X_{s}^{t, x, i ; u, v}, Y_{s}^{1, u, v}, Z_{s}^{1, u, v}, i, u_{s}, v_{s}\right) \mathrm{d} s-Z_{s}^{1, u, v} \mathrm{~d} B_{s}, \\
Y_{t+\delta}^{1, u, v}=0
\end{array}\right.
$$

where the process $X_{s}^{t, x, i ; u, v}$ has been introduced by (2), $(t, x, i) \in[0, T] \times R^{n} \times \mathscr{M}$ is regarded as the initial state, $X_{s}$ is the value of $X$ at time $s$, and $u \in U_{t, t+\delta}, v \in V_{t, t+\delta}$.

We can characterize the solution process $Y^{1, u, v}$ as follows.

Lemma 8. For any $s \in[t, t+\delta], \quad(t, x) \in[0, T] \times R^{n}$, $\phi \in C_{l, b}^{3}\left([0, T] \times R^{n}\right)$, we define $\varphi \in\left(C\left([0, T] \times R^{n}\right)\right)^{m}$ by $\varphi(\cdot, \cdot, i)=\phi(\cdot)$, and $\varphi(\cdot, \cdot, k)=W(\cdot, \cdot, k)$, and for $k \neq i$, we have the following relationship:

$$
E\left[Y_{s}^{1, u, v} \mid \mathscr{F}_{t}\right]=E\left[G_{s, t+\delta}^{t, x, i ; u, v}\left[\varphi\left(t+\delta, X_{t+\delta}^{t, x, i ; u, v}, \theta_{t+\delta}^{t, i}\right)\right]-\varphi\left(s, X_{s}^{t, x, i ; u, v}, \theta_{s}^{t, i}\right) \mid \mathscr{F}_{t}\right], \quad P-\text { a.s. }
$$

Proof. $G_{s, t+\delta}^{t, x, i ; u, v}\left[\varphi\left(t+\delta, X_{t+\delta}^{t, x, i ; u, v}, \theta_{t+\delta}^{t, i}\right)\right]$ is defined with the help of the solution of the BSDE:

$$
\left\{\begin{array}{l}
-\mathrm{d} Y_{s}^{u, v}=f\left(s, X_{s}^{t, x, i ; u, v}, Y_{s}^{u, v}, Z_{s}^{u, v}, i, u_{s}, v_{s}\right) \mathrm{d} s-Z_{s}^{u, v} \mathrm{~d} B_{s}, \\
Y_{t+\delta}^{u, v}=\varphi\left(t+\delta, X_{t+\delta}^{t, x, i ; u, v}, \theta_{t+\delta}^{t, i}\right), \quad s \in[t, t+\delta],
\end{array}\right.
$$

by the following formula:

$$
G_{s, t+\delta}^{t, x, i ; u, v}\left[\varphi\left(t+\delta, X_{t+\delta}^{t, x, i ; u, v}, \theta_{t+\delta}^{t, i}\right)\right]=Y_{s}^{u, v} .
$$

Thus, we only need to prove $Y_{s}^{u, v}-\varphi\left(s, X_{s}^{t, x, i ; u, v}\right.$, $\left.\theta_{s}^{t, i}\right)=Y_{s}^{1, u, v}$. We have

$$
E\left[Y_{s}^{u, v}-\varphi\left(s, X_{s}^{t, x, i ; u, v}, \theta_{s}^{t, i}\right) \mid \mathscr{F}_{t}\right]=E\left[Y_{s}^{1, u, v} \mid \mathscr{F}_{t}\right],
$$

by applying Dynkin's formula to $\varphi\left(s, X_{s}^{t, x, i ; u, v}, \theta_{s}^{t, i}\right)$. And for $s=t+\delta$,

$$
E\left[Y_{t+\delta}^{u, v}-\varphi\left(t+\delta, X_{t+\delta}^{t, x, i ; u, v}, \theta_{t+\delta}^{t, i}\right) \mid \mathscr{F}_{t}\right]=E\left[Y_{t+\delta}^{1, u, v} \mid \mathscr{F}_{t}\right]=0
$$

Therefore, for any $s \in[t, t+\delta]$, we get the desired result.
We consider the following simple BSDE in which $X_{s}^{t, x, i ; u, v}$ is replaced by its deterministic initial value $x$ :

$$
\left\{\begin{array}{l}
-\mathrm{d} Y_{s}^{2, u, v}=F\left(s, x, Y_{s}^{2, u, v}, Z_{s}^{2, u, v}, i, u_{s}, v_{s}\right) \mathrm{d} s-Z_{s}^{2, u, v} \mathrm{~d} B_{s}, \\
Y_{t+\delta}^{2, u, v}=0, \quad s \in[t, t+\delta] .
\end{array}\right.
$$

Then, we have the following lemma.

Lemma 9. There is a constant $C>0$ independent of the control processes $u, v$ and of $\delta>0$, such that for every $u \in U_{t, t+\delta}, v \in V_{t, t+\delta}$,

$$
E\left[\left|Y_{t}^{1, u, v}-Y_{t}^{2, u, v}\right| \mid \mathscr{F}_{t}\right] \leq C \delta^{(3 / 2)}, \quad P-\text { a.s. }
$$

Proof. From Lemma 4, we have the existence of some constant $C>0$ such that

$$
E\left[\sup _{s \in[t, T]}\left|X_{s}^{t, x, i ; u, v}\right|^{2} \mid \mathscr{F}_{t}\right] \leq C\left(1+|x|^{2}\right),
$$


combined with

$$
\begin{aligned}
E\left[\sup _{s \in[t, t+\delta]}\left|X_{s}^{t, x, i ; u, v}-x\right|^{2} \mid \mathscr{F}_{t}\right] \leq & 2 E\left[\sup _{s \in[t, t+\delta]}\left|\int_{t}^{s} b\left(r, X_{r}^{t, x, i ; u, v}, i, u_{r}, v_{r}\right) \mathrm{d} r\right|^{2} \mid \mathscr{F}_{t}\right] \\
& +2 E\left[\sup _{s \in[t, t+\delta]}\left|\int_{t}^{s} \sigma\left(r, X_{r}^{t, x, i ; u, v}, i, u_{r}, v_{r}\right) \mathrm{d} B_{r}\right|^{2} \mid \mathscr{F}_{t}\right],
\end{aligned}
$$

and we have

$$
E\left[\sup _{s \in[t, t+\delta]}\left|X_{s}^{t, x, i ; u, v}-x\right|^{2} \mid \mathscr{F}_{t}\right] \leq C \delta .
$$

$$
\begin{aligned}
\eta_{1} & =\eta_{2}=0, \\
f(s, y, z) & =F\left(s, X_{s}^{t, x, i ; u, v}, y, z, i, u_{s}, v_{s}\right), \\
\varphi_{1}(s) & =0 \\
\varphi_{2}(s) & =F\left(s, x, Y_{s}^{2, u, v}, Z_{s}^{2, u, v}, i, u_{s}, v_{s}\right)-F\left(s, X_{s}^{t, x, i ; u, v}, Y_{s}^{2, u, v}, Z_{s}^{2, u, v}, i, u_{s}, v_{s}\right) .
\end{aligned}
$$

It is easy to know that $g$ is Lipschitz with respect to $(y, z)$, and $\left|\varphi_{2}(s)\right| \leq C\left(1+|x|^{2}\right)\left(\left|X_{s}^{t, x, i ; i, v}-x\right|+\mid X_{s}^{t, x, i ; u, v}-x\right.$ $\left.\left.\right|^{3}\right)$ for $s \in[t, t+\delta], \quad(t, x, i) \in[0, T) \times R^{n} \times \mathscr{M}, u \in U_{t, t+\delta}$, $v \in V_{t, t+\delta}$. Thus,

$$
\begin{gathered}
E\left[\int_{t}^{t+\delta}\left(\left|Y_{s}^{1, u, v}-Y_{s}^{2, u, v}\right|^{2}+\left|Z_{s}^{1, u, v}-Z_{s}^{2, u, v}\right|^{2}\right) \mathrm{d} s \mid \mathscr{F}_{t}\right] \\
\leq C E\left[\int_{t}^{t+\delta} \rho_{0}^{2}\left(\left|X_{s}^{t, x, i ; u, v}-x\right|\right) \mathrm{d} s \mid \mathscr{F}_{t}\right] \\
\quad \leq C \delta E\left[\sup _{s \in[t, t+\delta]} \rho_{0}^{2}\left(\left|X_{s}^{t, x i ; u, v}-x\right|\right) \mid \mathscr{F}_{t}\right] \leq C \delta^{2},
\end{gathered}
$$

where $\rho_{0}(r)=\left(1+|x|^{2}\right)\left(r+r^{3}\right), r \geq 0$. Therefore,

$$
\begin{aligned}
E\left[\left|Y_{t}^{1, u, v}-Y_{t}^{2, u, v}\right| \mid \mathscr{F}_{t}\right]= & E\left[\left|E\left(Y_{t}^{1, u, v}-Y_{t}^{2, u, v}\right)\right| \mathscr{F}_{t} \mid \mathscr{F}_{t}\right] \\
= & E\left[\mid E\left[\int _ { t } ^ { t + \delta } \left(F\left(s, X_{s}^{t, x, i, u, v}, Y_{s}^{1, u, v}, Z_{s}^{1, u, v}, i, u_{s}, v_{s}\right)\right.\right.\right. \\
& \left.\left.\left.-F\left(s, x, Y_{s}^{2, u, v}, Z_{s}^{2, u, v}, i, u_{s}, v_{s}\right) \mathrm{d} s \mid \mathscr{F}_{t}\right)\right]|| \mathscr{F}_{t}\right] \\
& \leq C E\left[\int_{t}^{t+\delta}\left[\rho_{0}\left(\left|X_{s}^{t, x, i ; u, v}-x\right|\right)+\left|Y_{s}^{1, u, v}-Y_{s}^{2, u, v}\right|+\left|Z_{s}^{1, u, v}-Z_{s}^{2, u, v}\right| \mathrm{d} s\right] \mid \mathscr{F}_{t}\right] \\
& \leq C E\left[\int_{t}^{t+\delta} \rho_{0}\left(\left|X_{s}^{t, x, i ; u, v}-x\right|\right) \mathrm{d} s \mid \mathscr{F}_{t}\right]+C \delta^{(1 / 2)} E\left[\int_{t}^{t+\delta}\left|Y_{s}^{1, u, v}-Y_{s}^{2, u, v}\right|^{2} \mathrm{~d} s \mid \mathscr{F}_{t}\right]^{(1 / 2)} \\
& +C \delta^{(1 / 2)} E\left[\int_{t}^{t+\delta}\left|Z_{s}^{1, u, v}-Z_{s}^{2, u, v}\right|^{2} \mid \mathscr{F}_{t}\right]^{(1 / 2)} \\
& \leq C \delta^{(3 / 2)} .
\end{aligned}
$$


The proof is complete.

Lemma 10. Let $Y_{0}(\cdot)$ be the solution of the following ordinary differential equation:

$$
\left\{\begin{array}{l}
-\mathrm{d} Y_{0}(s)=F_{0}\left(s, x, Y_{0}(s), 0, i\right), \quad s \in[t, t+\delta], \\
Y_{0}(t+\delta)=0,
\end{array}\right.
$$

where

$$
F_{0}(s, x, y, z, i)=\sup _{u \in U} \inf _{v \in V} F(s, x, y, z, i ; u, v) .
$$

Then, $P-$ a.s.,

$$
\underset{u \in U_{t, t+\delta}}{\operatorname{essup}} \underset{v \in V_{t, t+\delta}}{\operatorname{essinf}} E\left[Y_{t}^{2, u, v} \mid \mathscr{F}_{t}\right]=E\left[Y_{0}(t) \mid \mathscr{F}_{t}\right] \text {. }
$$

Proof. We first introduce the function

$$
F_{1}(s, x, y, z, i, u)=\inf _{v \in V} F(s, x, y, z, i, u, v),
$$

where $(s, x, y, z, i, u) \in[0, T] \times R^{n} \times R \times R^{d} \times \mathscr{M} \times U$. And we consider the following equation: for $u \in U_{t, t+\delta}$,

$$
\left\{\begin{array}{l}
-\mathrm{d} Y_{s}^{3, u}=F_{1}\left(s, x, Y_{s}^{3, u}, Z_{s}^{3, u}, i, u_{s}\right) \mathrm{d} s-Z_{s}^{3, u} \mathrm{~d} B_{s}, \\
\left.Y_{t+\delta}^{3, u}=0, \quad s \in t, t+\delta\right] .
\end{array}\right.
$$

Since $F_{1}\left(s, x, y, z, i, u_{s}\right)$ is Lipschitz in $(y, z)$, for every $u \in U_{t, t+\delta}$, there exists a unique solution $\left(Y^{3, u}, Z^{3, u}\right)$ to $(74)$. Then, for every $u \in U_{t, t+\delta}$,

$$
E\left[Y_{t}^{3, u} \mid \mathscr{F}_{t}\right]=\underset{v \in V_{t, t+\delta}}{\operatorname{essinf}} E\left[Y_{t}^{2, u, v} \mid \mathscr{F}_{t}\right], \quad P-\text { a.s. }
$$

In fact, from Lemma 2 and the definition of $F_{1}$, for every $u \in U_{t, t+\delta}$, we have

$$
E\left[Y_{t}^{3, u} \mid \mathscr{F}_{t}\right] \leq \operatorname{essinf}_{v \in V_{t, t+\delta}} E\left[Y_{t}^{2, u, v} \mid \mathscr{F}_{t}\right], \quad P-\text { a.s. }
$$

Moreover, there exists a measurable function $v^{4}:[t, T] \times$ $R^{n} \times R \times R^{d} \times \mathscr{M} \times U \longrightarrow V$ such that

$$
F_{1}(s, x, y, z, i, u)=F\left(s, x, y, z, i, u, v^{4}(s, x, y, z, i, u)\right) \text { for any } s, x, y, z, u \text {. }
$$

Let $\widetilde{v}_{s}^{4}:=v^{4}\left(s, x, Y_{s}^{3, u}, Z_{s}^{3, u}, i, u_{s}\right), s \in[t, t+\delta]$, and we observe that $\widetilde{v}^{4} \in V_{t, t+\delta}$, and

$$
F_{1}\left(s, x, Y_{s}^{3, u}, Z_{s}^{3, u}, i, u_{s}\right)=F\left(s, x, Y_{s}^{3, u}, Z_{s}^{3, u}, i, u_{s}, \widetilde{v}_{s}^{4}\right), \quad s \in[t, t+\delta] .
$$

Thus, from the uniqueness of the solution of the BSDE, it follows that $\left(Y^{3, u}, Z^{3, u}\right)=\left(Y^{2, u, \widetilde{v}^{4}}, Z^{2, u, \widetilde{v}^{4}}\right)$ and $E\left[Y_{t}^{3, u} \mid \mathscr{F}_{t}\right]=E\left[Y_{t}^{2, u, \widetilde{v}^{4}} \mid \mathscr{F}_{t}\right], P-$ a.s., for every $u \in U_{t, t+\delta}$. Then, for all $u \in U_{t, t+\delta}$,

$$
E\left[Y_{t}^{3, u} \mid \mathscr{F}_{t}\right]=\underset{v \in V_{t, t+\delta}}{\operatorname{essinf}} E\left[Y_{t}^{2, u, v} \mid \mathscr{F}_{t}\right], \quad P-\text { a.s. }
$$

Then, since $F_{0}(s, x, y, z, i)=\sup _{u \in U_{t, t+\delta}} F_{1}(s, x, y, z, i, u)$, by a similar proof, we have

$$
\begin{aligned}
E\left[Y_{0}(t) \mid \mathscr{F}_{t}\right] & =\underset{u \in U_{t, t+\delta}}{\operatorname{essup}} E\left[Y_{t}^{3, u} \mid \mathscr{F}_{t}\right] \\
& =\operatorname{esssup}_{u \in U_{t, t+\delta}} \operatorname{essinf}_{v \in V_{t, t+\delta}} E\left[Y_{t}^{2, u, v} \mid \mathscr{F}_{t}\right], \quad P-\text { a.s. }
\end{aligned}
$$

It uses the fact that (70) can be considered as a BSDE with the solution $\left(Y_{s}, Z_{s}\right)=\left(Y_{0}(s), 0\right)$. So, the proof is complete.

Lemma 11. For all $u \in U_{t, t+\delta}, v \in V_{t, t+\delta}$, we have

$$
E\left[\int_{t}^{t+\delta}\left|Y_{s}^{2, u, v}\right| \mathrm{d} s \mid \mathscr{F}_{t}\right]+E\left[\int_{t}^{t+\delta}\left|Z_{s}^{2, u, v}\right| \mathrm{d} s \mid \mathscr{F}_{t}\right] \leq C \delta^{(3 / 2)}, \quad P-\text { a.s. }
$$

where the constant $C$ is independent of the control processes $u$, $v$ and of $\delta>0$.
Proof. Since $F(s, x, \cdot, \cdot, i, u, v)$ has a linear growth in $(y, z)$, uniformly in $(u, v)$, we get from Lemma 2 for some constant $C$ independent of $\delta$ and the controls $u, v$,

$$
\left|Y_{s}^{2, u, v}\right|^{2} \leq C \delta, E\left[\int_{s}^{t+\delta}\left|Z_{r}^{2, u, v}\right| \mathrm{d} r \mid \mathscr{F}_{s}\right] \leq C \delta, \quad s \in[t, t+\delta] .
$$

Moreover, from equation (62),

$$
\begin{aligned}
\left|Y_{s}^{2, u, v}\right| & \leq E\left[\int_{s}^{t+\delta} F\left(r, x, Y_{r}^{2, u, v}, Z_{r}^{2, u, v}, i, u_{r}, v_{r}\right)|\mathrm{d} r| \mathscr{F}_{s}\right] \\
& \leq C E\left[\int_{s}^{t+\delta}\left(1+|x|^{2}+\left|Y_{r}^{2, u, v}\right|+\left|Z_{r}^{2, u, v}\right|\right) \mathrm{d} r \mid \mathscr{F}_{s}\right] \\
& \leq C \delta+C \delta^{(1 / 2)}\left(E\left[\int_{s}^{t+\delta}\left|Z_{r}^{2, u, v}\right|^{2} \mathrm{~d} r \mid \mathscr{F}_{s}\right]\right)^{(1 / 2)} \\
& \leq C \delta, \quad P-\text { a.s. } s \in[t, t+\delta],
\end{aligned}
$$

and since

$$
\int_{t}^{t+\delta} Z_{s}^{2, u, v} \mathrm{~d} B_{s}=\int_{t}^{t+\delta} F\left(s, x, Y_{s}^{2, u, v}, Z_{s}^{2, u, v}, i, u_{s}, v_{s}\right) \mathrm{d} s-Y_{t}^{2, u, v},
$$

we get

$$
E\left[\int_{t}^{t+\delta}\left|Z_{s}^{2, u, v}\right|^{2} \mathrm{~d} s \mid \mathscr{F}_{t}\right] \leq C \delta^{2} .
$$

Therefore, 


$$
\begin{aligned}
& E\left[\int_{t}^{t+\delta}\left|Y_{s}^{2, u, v}\right| \mathrm{d} s \mid \mathscr{F}_{t}\right]+E\left[\int_{t}^{t+\delta}\left|Z_{s}^{2, u, v}\right| \mathrm{d} s \mid \mathscr{F}_{t}\right] \\
& \quad \leq C \delta^{2}+C \delta^{(1 / 2)}\left(E\left[\int_{t}^{t+\delta}\left|Z_{s}^{2, u, v}\right|^{2} \mathrm{~d} r \mid \mathscr{F}_{t}\right]\right)^{(1 / 2)} \\
& \quad \leq C \delta^{(3 / 2)}, \quad P \text { - a.s. }
\end{aligned}
$$

\section{Proof of Theorem 1}

(i) It is easy to know that $W(T, x, i)=\Phi(x, i)$, $(x, i) \in R^{n} \times \mathscr{M}$. We first will prove $W$ is a viscosity supersolution. For fixed $i \in \mathscr{M}$, without loss of generality, we suppose that $\varphi(t, x, i)=W(t, x, i)$. According to Proposition 1,

$$
\begin{aligned}
\varphi(t, x, i) & =W(t, x, i) \\
& =\operatorname{essinf}_{\beta \in \mathscr{B}_{t, t+\delta}}^{\operatorname{esssup}} \in\left[G_{u \in U_{t, t+\delta}}^{t, x, i ; u, \beta(u)}\left[W\left(t+\delta, X_{t+\delta}^{t, x, i ; u, \beta(u)}, \theta_{t+\delta}^{t, i}\right)\right] \mid \mathscr{F}_{t}\right],
\end{aligned}
$$

where $0 \leq \delta \leq T-t$ and from $W \geq \varphi$ and the monotonicity property of $G_{t, t+\delta}^{t, x, i ; u, \beta(u)}[\cdot]$, we have

$$
\underset{\beta \in \mathscr{B}_{t, t+\delta}}{\operatorname{essinf}} \operatorname{esssup} E\left[\left(G_{t, t+\delta}^{t, x, i ; u, \beta(u)}\left[\varphi\left(t+\delta, X_{t, t+\delta}^{t, x, i ; u, \beta(u)}, \theta_{t+\delta}^{t, i}\right)-\varphi(t, x, i)\right]\right) \mid \mathscr{F}_{t}\right] \leq 0, \quad P-\text { a.s. }
$$

From Lemma 8,

$\underset{\beta \in \mathscr{B}_{t, t+\delta}}{\operatorname{essinf}} \operatorname{esssup} E\left[Y_{t}^{1, u, \beta(u)} \mid \mathscr{F}_{t}\right] \leq 0, \quad P$ - a.s.

Thus, from Lemma 9, we obtain

$\underset{\beta \in \mathscr{B}_{t, t+\delta}}{\operatorname{essinf}} \operatorname{esssup} E\left[Y_{t}^{2, u, \beta(u)} \mid \mathscr{F}_{t}\right] \leq C \delta^{(3 / 2)}, \quad P-$ a.s.
Then, since

$$
\underset{v \in V_{t, t+\delta}}{\operatorname{essinf}} E\left[Y_{t}^{2, u, v} \mid \mathscr{F}_{t}\right] \leq E\left[Y_{t}^{2, u, \beta(u)} \mid \mathscr{F}_{t}\right], \quad \beta \in \mathscr{B}_{t, t+\delta}
$$

we have

$$
\underset{u \in U_{t, t+\delta}}{\operatorname{esssup}} \operatorname{essinf} E\left[Y_{t, t+\delta}^{2, u, v} \mid \mathscr{F}_{t}\right] \leq \underset{\beta \in \mathscr{B}_{t, t+\delta}}{\operatorname{essinf}} \operatorname{esssup} E\left[Y_{t}^{2, u, \beta(u)} \mid \mathscr{F}_{t}\right] \leq C \delta^{(3 / 2)}, \quad P-\text { a.s, }
$$

and Lemma 10 implies $Y_{0}(t) \leq C \delta^{(3 / 2)}, P$ - a.s., where $Y_{0}$ is the unique solution of (70). Thus,

$$
\begin{aligned}
C \delta^{(1 / 2)} \geq & \frac{1}{\delta} Y_{0}(t)=\frac{1}{\delta} \int_{t}^{t+\delta} F_{0}\left(s, x, Y_{0}(s), 0, i\right) \mathrm{d} s, \quad \delta>0, \\
& \sup _{u \in U} \inf _{v \in V} F(t, x, 0,0, i, u, v)=F_{0}(t, x, 0,0, i) \leq 0,
\end{aligned}
$$

and from the definition of $F$, we see that $W$ is a viscosity supersolution of (7).

(ii) Now we prove $W$ is a viscosity subsolution. For fixed $i \in \mathscr{M}$, without loss of generality, we suppose that $\varphi(t, x, i)=W(t, x, i)$. We must prove that

$$
\sup _{u \in U} \inf _{v \in V} F(t, x, 0,0, i, u, v)=F_{0}(t, x, 0,0, i) \geq 0 .
$$

We suppose that this is not true. Then, there exists some $R>0$ such that

$$
F_{0}(t, x, 0,0, i)=\sup _{u \in U} \inf _{v \in V} F(t, x, 0,0, i, u, v) \leq-R<0,
$$

and we can find a measurable function $\psi: U \longrightarrow V$ such that

$$
F(t, x, 0,0, i, u, \psi(u)) \leq-\frac{3}{4} R \text { for all } u \in U .
$$

And since $F(\cdot, x, 0,0, i, \cdot, \cdot)$ is uniformly continuous on $[0, T] \times U \times V$, there exists some $0<Q \leq T-t$ such that for all $u \in U$ and $|s-t| \leq Q$, 


$$
F(s, x, 0,0, i, u, \psi(u)) \leq-\frac{1}{2} R
$$

$$
\begin{aligned}
\varphi(t, x, i) & =W(t, x, i) \\
& =\underset{\beta \in \mathscr{B}_{t, t+\delta}}{\operatorname{essinf}} \operatorname{essinf}_{u \in U_{t, t+\delta}} E\left[G_{t, t+\delta}^{t, x, i ; u, \beta(u)}\left[W\left(t+\delta, X_{t+\delta}^{t, x, i ; u, \beta(u)}, \theta_{t+\delta}^{t, i}\right)\right] \mid \mathscr{F}_{t}\right],
\end{aligned}
$$

and similar to (i), from $W \leq \varphi$ and the monotonicity property of $G_{t, t+\delta}^{t, x, i ; u, \beta(u)}[\cdot]$, we have

$$
\underset{\beta \in \mathscr{B}_{t, t+\delta}}{\operatorname{essinf}} \operatorname{essup} E\left[\left(G_{t, t+\delta}^{t, x, i ; u, \beta(u)}\left[\varphi\left(t+\delta, X_{t+\delta}^{t, x, i ; u, \beta(u)}, \theta_{t+\delta}^{t, i}\right)\right]-\varphi(t, x, i)\right) \mid \mathscr{F}_{t}\right] \geq 0, \quad P-\text { a.s. }
$$

Therefore, from Lemma 8,

$\underset{\beta \in \mathscr{B}_{t, t+\delta}}{\operatorname{essinf}} \operatorname{esssup} E\left[Y_{t}^{1, u, \beta(u)} \mid \mathscr{F}_{t}\right] \geq 0, \quad P-$ a.s.

Thus,

$$
\underset{u \in U_{t, t+\delta}}{\operatorname{esssup}} E\left[Y_{t}^{1, u, \psi(u)} \mid \mathscr{F}_{t}\right] \geq 0, \quad P-\text { a.s. }
$$

Here, by setting $\psi_{s}(u)(\omega)=\psi\left(u_{s}(\omega)\right), \quad(s, \omega) \in[t$, $T] \times \Omega$, we identify $\psi$ as an element of $\mathscr{B}_{t, t+\delta}$. Given any $\varepsilon_{\dot{\varepsilon}} 0$, we can choose $u^{\varepsilon} \in U_{t, t+\delta}$ such that $E\left[Y_{t}^{1, u^{\varepsilon}, \psi\left(u^{\varepsilon}\right)} \mid \mathscr{F}_{t}\right] \geq-\varepsilon \delta$. From Lemma 9, we get

$$
E\left[Y_{t}^{2, u^{\varepsilon}, \psi\left(u^{\varepsilon}\right)} \mid \mathscr{F}_{t}\right] \geq-C \delta^{(3 / 2)}-\varepsilon \delta, \quad P-\text { a.s. }
$$

Moreover, from (62),

$$
E\left[Y_{t}^{2, u^{\varepsilon}, \psi\left(u^{\varepsilon}\right)} \mid \mathscr{F}_{t}\right]=E\left[\int_{t}^{t+\delta} F\left(s, x, Y_{s}^{2, u^{\varepsilon}, \psi\left(u^{\varepsilon}\right)}, Z_{s}^{2, u^{\varepsilon}, \psi\left(u^{\varepsilon}\right)}, i, u_{s}^{\varepsilon}, \psi\left(u_{s}^{\varepsilon}\right)\right) \mathrm{d} s \mid \mathscr{F}_{t}\right] .
$$

We get from the Lipschitz property of $F$ in $(y, z),(99)$, and Lemma 11 that

$$
\begin{aligned}
& E\left[Y_{t}^{2, u^{\varepsilon}, \psi\left(u^{\varepsilon}\right)} \mid \mathscr{F}_{t}\right] \\
& \leq E\left[\int_{t}^{t+\delta}\left(C\left|Y_{s}^{2, u^{e}, \psi\left(u^{\varepsilon}\right)}\right|+C\left|Z_{s}^{2, u^{\varepsilon}, \psi\left(u^{\varepsilon}\right)}\right|+F\left(s, x, 0,0, i, u_{s}^{\varepsilon}, \psi_{s}\left(u^{\varepsilon}\right)\right)\right) \mathrm{d} s \mid \mathscr{F}_{t}\right] \\
& \leq C \delta^{(3 / 2)}-\frac{1}{2} R, \quad P \text { - a.s. }
\end{aligned}
$$


Letting $\delta \downarrow 0$ and then $\varepsilon \downarrow 0$, we get that $R \leq 0$, which induces a contradiction. Then,

$$
F_{0}(t, x, 0,0, i)=\sup _{u \in U} \inf _{v \in V} F(t, x, 0,0, i, u, v) \geq 0,
$$

and from the definition of $F$, we see that $W$ is a viscosity supersolution of (7). Therefore, from the above two steps, we derive that $W$ is a viscosity solution of (7).
Remark 2. We can also prove that $\mathrm{U}$ is a viscosity solution of (8) similarly by the method of Theorem 1.

We now study the uniqueness of the viscosity solution of Isaacs' equation (7):

$$
\left\{\begin{array}{l}
\frac{\partial}{\partial t} h(t, x, i)+H^{-}\left(t, x, i, h, D h, D^{2} h\right)+\sum_{k \neq i} q_{i k}[W(t, x, k)-W(t, x, i)]=0, \\
h(T, x, i)=\Phi(x, i),
\end{array}\right.
$$

where

$$
\begin{aligned}
H^{-}(t, x, i, p, A, u, v)= & \sup _{u \in U} \inf _{v \in V}\left\{\frac{1}{2} \operatorname{Tr}\left(\sigma \sigma^{T}(t, x, i, u, v) \cdot A\right)+\langle b(t, x, i, u, v), p\rangle\right. \\
& \left.+f\left(t, x, i, r, \sigma^{T}(t, x, i, u, v) p, u, v\right)\right\}
\end{aligned}
$$

for $\quad(t, x, i, r, p, A, u, v) \in[0, T] \times R^{n} \times \mathscr{M} \times R \times R^{n} \times R^{n \times n}$ $\times U \times V \longrightarrow R$. The functions $b, \sigma, f$, and $\Phi$ are still supposed to satisfy (A3) and (A4), respectively.
We will prove the uniqueness for (108) in the following space of continuous functions:

$$
\begin{aligned}
\Theta & =\left\{\phi \in C\left([0, T] \times R^{n}\right): \exists \tilde{A}>0\right. \text { such that } \\
& \left.\lim _{|x| \longrightarrow \infty} \phi(t, x) \exp \left\{-\tilde{A}\left[\log \left(\left(|x|^{2}+1\right)^{(1 / 2)}\right)\right]^{2}\right\}=0 \text { uniformly in } t \in[0, T]\right\} .
\end{aligned}
$$

This growth condition was introduced in [18]. And it was shown in [18] that this kind of growth condition is optimal for the uniqueness and can, in general, not be weakened. We adapt the idea developed in [18] to Isaacs' equation (108) to prove the uniqueness of the viscosity solution in $\Theta$. We only focus on equation (7) in $\Theta$, and results hold for equation (8) in a similar procedure. For the proof of the uniqueness theorem, we need some auxiliary lemmas. Denoting by $K$ a Lipschitz constant of $f(t, x, \cdot, \cdot, i, \cdot, \cdot)$, we have the following.

Lemma 12. Let $W^{1} \in \Theta$ be a viscosity subsolution and $W^{2} \in \Theta$ be a viscosity supersolution of (108). Then, the function $h:=W^{1}-W^{2}$ is a viscosity subsolution of the equation

$$
\left\{\begin{array}{l}
\frac{\partial}{\partial t} h(t, x)+\sup _{u, v}\left\{\frac{1}{2} \operatorname{Tr}\left(\sigma \sigma^{T}(t, x, i, u, v) D^{2} h\right)+D h \cdot b(t, x, i, u, v)+K|w|+\right. \\
K|D h \cdot \sigma(t, x, i, u, v)|\}+\sum_{k \neq i} q_{i k}[h(t, x, k)-h(t, x, i)]=0, \\
h(T, x, i)=0, \quad(t, x, i) \in[0, T) \times R^{n} \times \mathscr{M} .
\end{array}\right.
$$


The proof of this lemma follows directly from Lemma 3.7 in [18]; it is only slightly different.
Lemma 13. Let $W^{1} \in \Theta$ be a viscosity subsolution and $W^{2} \in \Theta$ be a viscosity supersolution of (108). Then, the function $\widetilde{h}:=\max _{i \in M}\left(W^{1}(t, x, i)-W^{2}(t, x, i)\right)$ is a viscosity subsolution of the equation

$$
\left\{\begin{array}{l}
\frac{\partial}{\partial t} \widetilde{h}(t, x)+\sup _{u, v, i}\left\{\frac{1}{2} \operatorname{Tr}\left(\sigma \sigma^{T}(t, x, i, u, v) D^{2} \widetilde{h}\right)+D \widetilde{h} \cdot b(t, x, i, u, v)+K|\widetilde{h}|+\right. \\
K|D \widetilde{h} \cdot \sigma(t, x, i, u, v)|\}=0 \\
\widetilde{h}(T, x)=0, \quad(t, x) \in[0, T) \times R^{n}
\end{array}\right.
$$

Proof. For $\left(t_{0}, x_{0}\right) \in[0, T) \times R^{n}$, without loss of generality, we suppose that $\widetilde{h}\left(t_{0}, x_{0}\right)=W^{1}\left(t_{0}, x_{0}, i\right)-W^{2}\left(t_{0}, x_{0}, i\right)$, i.e.,

$$
h\left(t_{0}, x_{0}, i\right) \geq h\left(t_{0}, x_{0}, k\right), \quad \forall k \neq i,
$$

$$
\sum_{k \neq i} q_{i k}\left[\left(h\left(t_{0}, x_{0}, k\right)-h\left(t_{0}, x_{0}, i\right)\right)\right] \leq 0
$$

Obviously, $\tilde{h}(T, x) \leq 0$, for all $x \in R^{n}$. Moreover, from (114), for all functions $\phi \in C_{l, b}^{3}\left([0, T] \times R^{n}\right)$,

where $h:=W^{1}-W^{2}$. Hence,

$$
\begin{aligned}
& \frac{\partial}{\partial t} \phi\left(t_{0}, x_{0}\right)+\sup _{u, v, i}\left\{\frac{1}{2} \operatorname{Tr}\left(\sigma \sigma^{T}\left(t_{0}, x_{0}, i, u, v\right) D^{2} \phi\right)+D \phi \cdot b\left(t_{0}, x_{0}, i, u, v\right)\right. \\
& \left.\quad+K|\phi|+K\left|D \phi \cdot \sigma\left(t_{0}, x_{0}, i, u, v\right)\right|\right\} \\
& \quad \geq \frac{\partial}{\partial t} \phi\left(t_{0}, x_{0}\right)+\sup _{u, v}\left\{\frac{1}{2} \operatorname{Tr}\left(\sigma \sigma^{T}\left(t_{0}, x_{0}, i, u, v\right) D^{2} \phi\right)+D \phi \cdot b\left(t_{0}, x_{0}, i, u, v\right)\right. \\
& \left.\quad+K|\phi|+K\left|D \phi \cdot \sigma\left(t_{0}, x_{0}, i, u, v\right)\right|\right\} \\
& \quad \geq \frac{\partial}{\partial t} \phi\left(t_{0}, x_{0}\right)+\sup _{u, v}\left\{\frac{1}{2} \operatorname{Tr}\left(\sigma \sigma^{T}\left(t_{0}, x_{0}, i, u, v\right) D^{2} \phi\right)+D \phi \cdot b\left(t_{0}, x_{0}, i, u, v\right)\right. \\
& \left.\quad+K|\phi|+K\left|D \phi \cdot \sigma\left(t_{0}, x_{0}, i, u, v\right)\right|\right\} \\
& \quad+\sum_{k \neq i} q_{i k}\left[\left(\phi\left(t_{0}, x_{0}, k\right)-\phi\left(t_{0}, x_{0}, i\right)\right)\right] .
\end{aligned}
$$

On the other hand, for any $\phi \in C_{l, b}^{3}\left([0, T] \times R^{n}\right)$ such that $\tilde{h}-\phi$ attains its local maximum value zero at $\left(t_{0}, x_{0}\right)$, we have that $h(\cdot, \cdot, i)-\phi(\cdot, i)$ attains its local maximum value zero at $\left(t_{0}, x_{0}\right)$. Since $h$ is a viscosity subsolution of (111), we have

$$
\begin{aligned}
& \frac{\partial}{\partial t} \phi\left(t_{0}, x_{0}\right)+\sup _{u, v}\left\{\frac{1}{2} \operatorname{Tr}\left(\sigma \sigma^{T}\left(t_{0}, x_{0}, i, u, v\right) D^{2} \phi\right)+D \phi \cdot b\left(t_{0}, x_{0}, i, u, v\right)\right. \\
& \left.\quad+K|\phi|+K\left|D \phi \cdot \sigma\left(t_{0}, x_{0}, i, u, v\right)\right|\right\} \\
& \quad+\sum_{k \neq i} q_{i k}\left[\left(\phi\left(t_{0}, x_{0}, k\right)-\phi\left(t_{0}, x_{0}, i\right)\right)\right] \geq 0 .
\end{aligned}
$$


Consequently,

$$
\begin{aligned}
& \frac{\partial}{\partial t} \phi\left(t_{0}, x_{0}\right)+\sup _{u, v, i}\left\{\frac{1}{2} \operatorname{Tr}\left(\sigma \sigma^{T}\left(t_{0}, x_{0}, i, u, v\right) D^{2} \phi\right)+D \phi \cdot b\left(t_{0}, x_{0}, i, u, v\right)\right. \\
& \left.\quad+K|\phi|+K\left|D \phi \cdot \sigma\left(t_{0}, x_{0}, i, u, v\right)\right|\right\} \geq 0
\end{aligned}
$$
(112).

It means that $\tilde{h}$ is a viscosity subsolution of equation

Similar to the proof of Theorem 6.1 in [8], we can prove that $\widetilde{h} \leq 0$, i.e., $W^{1}(t, x, i) \leq W^{2}(t, x, i)$. Then, we have the uniqueness theorem.

Theorem 2. Under the assumptions (A3) and (A4), the viscosity solution of (108) is unique in $\Theta$.

Remark 3. Since the lower value function $W$ is of at most linear growth, it belongs to $\Theta$, and it is easy to know that $W$ is the unique viscosity solution in $\Theta$ of (108). And we can prove that the upper value function $U$ is the unique viscosity solution in $\Theta$ of (8).

Remark 4. If Isaacs' condition holds, that is, if for any $(t, x, i, r, p) \in[0, T] \times R^{n} \times \mathscr{M} \times R \times R^{n}, H^{+}(t, x, i, r, p, A$, $u, v)=H^{-}(t, x, i, r, p, A, u, v)$, then (8) and (108) coincide, i.e., (7) and (8) coincide, and from the uniqueness in $\Theta$ of viscosity solution, it follows that $W=U$ which means the associated SDG has a value.

\section{Data Availability}

No data were used in this study.

\section{Conflicts of Interest}

The authors declare that they have no conflicts of interest.

\section{Acknowledgments}

This study was supported by the National Natural Science Foundation of China (nos. 12071292 and 11871121) and the Natural Science Foundation of Zhejiang Province (no. LY21A010001).

\section{References}

[1] S. Lv, "Two-player zero-sum stochastic differential games with regime switching," Automatica, vol. 114, p. 108819, 2020.

[2] Q. Zhang, "Stock trading: an optimal selling rule," SIAM Journal on Control and Optimization, vol. 40, no. 1, pp. 64-87, 2001.

[3] Q. Zhang and X. Y. Zhou, "Valuation of stock loans with regime switching," SIAM Journal on Control and Optimization, vol. 48, no. 3, pp. 1229-1250, 2009.

[4] E. Pardoux and S. G. Peng, "Adapted solution of a backward stochastic differential equation," Systems \& Control Letters, vol. 14, no. 1, pp. 55-61, 1990.
[5] S. Peng, "A generalized dynamic programming principle and Hamilton-Jacobi-Bellman equation," Stochastics and Stochastic Reports, vol. 38, no. 2, pp. 119-134, 1992.

[6] S. Hamadène and J. P. Lepeltier, "Zero-sum stochastic differential games and backward equations," Systems \& Control Letters, vol. 24, no. 4, pp. 259-263, 1995.

[7] S. Hamadène, J.-P. Lepeltier, and S. G. Peng, "BSDEs with continuous coefficients and stochastic differential games," in Backward Stochastic Differential Equations, pp. 115-128, Longman, Harlow, UK, 1997.

[8] R. Buckdahn and J. Li, "Stochastic differential games and viscosity solutions of Hamilton-Jacobi-Bellman-Isaacs equations," SIAM Journal on Control and Optimization, vol. 47, no. 1, pp. 444-475, 2008.

[9] S. Peng, "A general stochastic maximum principle for optimal control problems," SIAM Journal on Control and Optimization, vol. 28, no. 4, pp. 966-979, 1990.

[10] S. G. Peng, "Probabilistic interpretation for systems of quasilinear parabolic partial difierential equations," Stochastics and Stochastics Reports, vol. 37, p. 10, 1991.

[11] N. El. Karoui, S. G. Peng, and M. C. Quenez, "Backward stochastic differential equations in finance," Mathematical Finance, vol. 7, no. 1, pp. 1-71, 1997.

[12] R. M. Xu and F. Zhang, " $\epsilon$-nash mean-field games for general linear-quadratic systems with applications," Automatica, vol. 114, Article ID 108835, 2020.

[13] X. F. Yi, R. W. Guo, and Y. Qi, "Stabilization of chaotic systems with both uncertainty and disturbance by the udebased control method," IEEE Access, vol. 8, pp. 62471-62477, 2020.

[14] Y. Y. Liu, T. Hou, and F. Q. Deng, "Stability for discrete-time uncertain systems with infinite markov jump and time-delay," Science China Information Sciences, vol. 64, Article ID 152202, 2021.

[15] R. W. Guo and Y. Qi, "Partial anti-synchronization in a class of chaotic and hyper-chaotic systems," IEEE Access, vol. 9, pp. 46303-46312, 2021.

[16] W. Fleming and P. Souganidis, "On the existence of value functions of two-player, zero-sum stochastic differential games," Indiana University Mathematics Journal, vol. 38, no. 2, pp. 293-314, 1989.

[17] S. G. Peng, "Backward stochastic differential equations-stochatic optimization theory and viscosity solutions of HJB equations," in Stochastic Analysis, Science Press, Beijing, China, 1997.

[18] G. Barles, R. Buckdahn, and E. Pardoux, "Backward stochastic differential equations and integral-partial differential equations," Stochastics and Stochastic Reports, vol. 60, no. 1-2, pp. 57-83, 1997. 